\title{
Oscillating simply connected wandering domains
}

\author{
VASILIKI EVDORIDOU(D, PHILIP J. RIPPON ${ }^{\circledR}$ and GWYNETH M. STALLARD \\ School of Mathematics and Statistics, The Open University, \\ Walton Hall, Milton Keynes MK7, 6AA, UK \\ (e-mail:vasiliki.evdoridou@open.ac.uk,phil.rippon@open.ac.uk, \\ gwyneth.stallard@open.ac.uk)
}

(Received 26 February 2021 and accepted in revised form 5 December 2021)

\begin{abstract}
Although detailed descriptions of the possible types of behaviour inside periodic Fatou components have been known for over 100 years, a classification of wandering domains has only recently been given. Recently, simply connected wandering domains were classified into nine possible types and examples of escaping wandering domains of each of these types were constructed. Here we consider the case of oscillating wandering domains, for which only six of these types are possible. We use a new technique based on approximation theory to construct examples of all six types of oscillating simply connected wandering domains. This requires delicate arguments since oscillating wandering domains return infinitely often to a bounded part of the plane. Our technique is inspired by that used by Eremenko and Lyubich to construct the first example of an oscillating wandering domain, but with considerable refinements which enable us to show that the wandering domains are bounded, to specify the degree of the mappings between wandering domains and to give precise descriptions of the dynamical behaviour of these mappings.
\end{abstract}

Key words: oscillating wandering domains, internal dynamics, Approximation Theory 2020 Mathematics Subject Classification: 37F10 (Primary); 30D05 (Secondary)

\section{Introduction}

Let $f$ be a transcendental entire function. We consider the iterates of $f$, which we denote by $f^{n}, n \geq 1$. The complex plane is divided into two sets: the Fatou set, $F(f)$, where the iterates $\left(f^{n}\right)$ form a normal family in a neighbourhood of every point, and its complement, the Julia set $J(f)$. An introduction to the theory of iteration of transcendental entire and meromorphic functions can be found in [Ber93].

The Fatou set is open and consists of connected components, which are called Fatou components. Fatou components can be periodic, preperiodic or wandering domains. A Fatou component $U$ is called a wandering domain if $f^{n}(U) \cap f^{m}(U)=\emptyset$, for all 
$n \neq m$. Although Sullivan showed in [Sul85] that rational maps have no wandering domains, transcendental entire functions can have wandering domains. The first example of such a function was given by Baker [Bak76] who proved that a certain entire function given by an infinite product has a multiply connected wandering domain. Several examples of simply connected wandering domains have been constructed since then (see, for example, [Her84, p. 104], [Sul85, p. 414], [Bak84, pp. 564 and 567], [Dev90, p. 222], [EL87, Examples 1 and 2], [FH06]).

In [BRS13] the authors gave a complete description of the dynamical behaviour in multiply connected wandering domains. Recently, in $[\mathrm{BEF}+21]$ the authors gave a detailed classification of simply connected wandering domains in terms of the hyperbolic distance between orbits of points and in terms of convergence to the boundary. More specifically, they classified simply connected wandering domains into contracting, semi-contracting and eventually isometric depending on whether, for almost all pairs of points in the wandering domain, the hyperbolic distances between the orbits of these points, tend to 0 , decrease but do not tend to 0 , or are eventually constant, respectively (see Theorem 5.1). In terms of convergence to the boundary, the orbits of all points stay away from the boundary, come arbitrarily close to the boundary but do not converge to it (bungee), or converge to the boundary (see Theorem 5.2). These two classifications give nine possible types of simply connected wandering domains. Using a new technique, based on approximation theory, they show that all of these nine possible types are indeed realizable.

All the examples constructed in $[\mathrm{BEF}+21]$ were escaping wandering domains. Hence it is natural to ask whether there exist oscillating wandering domains of all nine types. (It remains a major open question as to whether it is possible to have wandering domains of bounded orbit.) A wandering domain $U$ is called oscillating if there exist $\left(n_{k}\right),\left(m_{k}\right)$ such that $f^{n_{k}}(z) \rightarrow \infty$ and $\left(f^{m_{k}}(z)\right)$ stays bounded for all $z \in U$. Since in oscillating wandering domains the iterates of $f$ have finite limit points in $J(f)$, as well as $\infty$, it is impossible for the orbit of a point in such a wandering domain to stay away from the boundary. Thus three of the nine possible types are not realizable. In this paper we show that the remaining six possible types of oscillating wandering domains are all realizable.

The first transcendental entire function with oscillating wandering domains was given by Eremenko and Lyubich in [EL87]; this was also the first application of approximation theory in complex dynamics. The authors used sequences of discs and half-annuli and a model function which was constant on the half-annuli and a translation on the discs. This model function was approximated on the closure of every disc and half-annulus by a transcendental entire function using an extended version of Runge's approximation theorem. Their technique did not show though whether their wandering domains are bounded or not, and did not give information on the degree of the entire function on each of the wandering domains.

Motivated by the construction in [EL87], we adapt the new techniques from [BEF+21] to construct bounded oscillating wandering domains, which, moreover, have the property that the degree of $f$ on each of the wandering domains is equal to that of our model map. We state and prove our main construction theorem in $\S 3$.

It is worth pointing out that, in order for the wandering domains to be oscillating, the set-up needs to be much more complicated than that used for escaping wandering domains 
in $[\mathrm{BEF}+21]$. Although some of the building blocks of our proof are similar to those used in the analogous result for escaping wandering domains, the proof here requires several additional techniques. In particular, great care has to be taken over the accumulating errors in the approximation, as each of the discs on which the approximation takes place contains infinitely many domains in the orbit of the wandering domain.

In $\$ 5$ we use the main construction theorem to construct all six types of oscillating wandering domains, proving the following result. This requires several preliminary results concerning Blaschke products which we prove in $\S 4$.

THEOREM 1.1. For each of the six possible types of simply connected oscillating wandering domains, there exists a transcendental entire function with a bounded, simply connected oscillating wandering domain of that type.

Oscillating wandering domains for functions in the Eremenko-Lyubich class $\mathcal{B}$ have been constructed, first by Bishop in [Bis15], using the novel technique of quasiconformal folding, and more recently in [Boc21, FJL19, MPS20]. It would be interesting to see whether their methods can be adapted to classify the resulting wandering domains as one of the six possible types described above.

\section{Preliminary results}

In this section we give some existing results which are used in the proof of the main construction theorem. The following theorem, which is $[\mathrm{BEF}+\mathbf{2 1}$, Theorem D], plays a key role in the proof. We say that a curve $\sigma$ surrounds a curve $\sigma^{\prime}$ if $\sigma^{\prime}$ is contained in a bounded complementary component of $\sigma$. Also, for two compact sets $S, S^{\prime}$ we say that $S$ lies exterior to $S^{\prime}$ if $S$ is contained in the unbounded complementary component of $S^{\prime}$.

THEOREM 2.1. Let $f$ be a transcendental entire function and suppose that there exist Jordan curves $\gamma_{n}$ and $\Gamma_{n}, n \geq 0$, compact sets $L_{k}, k \geq 0$, and a bounded domain D such that:

(a) $\Gamma_{n}$ surrounds $\gamma_{n}$, for $n \geq 0$;

(b) the sets $\Gamma_{n}, n \geq 0, L_{k}, k \geq 0$, and $\bar{D}$ all lie exterior to each other;

(c) $\gamma_{n+1}$ surrounds $f\left(\gamma_{n}\right)$, for $n \geq 0$;

(d) $f\left(\Gamma_{n}\right)$ surrounds $\Gamma_{n+1}$, for $n \geq 0$;

(e) $f\left(\bar{D} \cup \bigcup_{k \geq 0} L_{k}\right) \subset D$;

(f) there exists $n_{k} \rightarrow \infty$ such that

$$
\max \left\{\operatorname{dist}\left(z, L_{k}\right): z \in \Gamma_{n_{k}}\right\}=o\left(\operatorname{dist}\left(\gamma_{n_{k}}, \Gamma_{n_{k}}\right)\right) \quad \text { as } k \rightarrow \infty .
$$

Then there exists an orbit of simply connected wandering domains $U_{n}$ such that $\overline{\text { int } \gamma_{n}} \subset$ $U_{n} \subset$ int $\Gamma_{n}$, for $n \geq 0$.

Moreover, if there exists $z_{n} \in$ int $\gamma_{n}$ such that both $f\left(\gamma_{n}\right)$ and $f\left(\Gamma_{n}\right)$ wind $d_{n}$ times around $f\left(z_{n}\right)$, then $f: U_{n} \rightarrow U_{n+1}$ has degree $d_{n}$, for $n \geq 0$.

In order to obtain the transcendental entire function with the required properties, we consider an analytic function which is our model function and then apply the following 
result which is an extension of the well-known Runge approximation theorem and was the Main Lemma in [EL87].

LEMMA 2.2. Let $\left(E_{n}\right)$ be a sequence of compact subsets of $\mathbb{C}$ with the following properties:

(i) $\mathbb{C} \backslash E_{n}$ is connected, for $n \geq 0$;

(ii) $E_{n} \cap E_{m}=\emptyset$, for $n \neq m$;

(iii) $\min \left\{|z|: z \in E_{n}\right\} \rightarrow \infty$ as $n \rightarrow \infty$.

Suppose $\psi$ is holomorphic on $E=\bigcup_{n=0}^{\infty} E_{n}$. For $n \geq 0$, let $\varepsilon_{n}>0$ and let $z_{n} \in E_{n}$. Then there exists an entire function $f$ satisfying, for $n \geq 0$,

$$
\begin{gathered}
|f(z)-\psi(z)|<\varepsilon_{n} \quad \text { for } z \in E_{n} \\
f\left(z_{n}\right)=\psi\left(z_{n}\right), \quad f^{\prime}\left(z_{n}\right)=\psi^{\prime}\left(z_{n}\right) .
\end{gathered}
$$

Remark 2.3. We note that if the sets $E_{n}$ are each real-symmetric (that is, $\overline{E_{n}}=E_{n}$ ), the function $\psi$ is real-symmetric in $E$ (that is, $\overline{\psi(\bar{z})}=\psi(z)$, for $z \in E$ ), and the points $z_{n}$, $n \geq 0$, are all real, then we can take the entire function $f$ to be real-symmetric on $\mathbb{C}$. Indeed, if $f$ satisfies the conclusions of Lemma 2.2, then $g(z)=\frac{1}{2}(f(z)+\overline{f(\bar{z})})$ is real-symmetric and entire, and satisfies the conclusions of Lemma 2.2.

We also need the following result, which is a version of [EL87, Lemma 2].

LEMMA 2.4. Let $g$ be an analytic function in the disc $\{z:|z|<R\}$ such that $g(0)=$ $g^{\prime}(0)=0$ and $|g(z)|<\epsilon R$ for $|z|<R$ and some $\epsilon<1 / 4$. Then

$$
|g(z)| \leq \frac{\epsilon}{R}|z|^{2} \quad \text { for }|z|<R .
$$

Finally, we need the following lemma about hyperbolic distances in discs, which is $\left[\mathrm{BEF}+21\right.$, Lemma 5.2]. Here we define $D_{r}=D(0, r)$ and $\mathbb{D}=D_{1}$.

Lemma 2.5. Suppose that $0<s<r<1<R$ and set

$$
c(s, R)=\frac{1-s^{2}}{R-s^{2} / R}, \quad D_{r}=D(0, r) \quad \text { and } \quad D_{R}=D(0, R) .
$$

If $|z|,|w| \leq s$, then

$$
\operatorname{dist}_{D_{R}}(z, w)=\operatorname{dist}_{\mathbb{D}}(z / R, w / R) \geq c(s, R) \operatorname{dist}_{\mathbb{D}}(z, w)
$$

and

$$
\operatorname{dist}_{D_{r}}(z, w)=\operatorname{dist}_{\mathbb{D}}(z / r, w / r) \leq \frac{1}{c(s / r, 1 / r)} \operatorname{dist}_{\mathbb{D}}(z, w) .
$$

Also $0<c(s, R)<1$, and if the variables $s, r$ and $R$ satisfy in addition

$$
1-r=o(1-s) \quad \text { as } s \rightarrow 1 \quad \text { and } \quad R-1=O(1-r) \quad \text { as } r \rightarrow 1 \text {, }
$$

then

$$
c(s, R) \rightarrow 1 \text { as } s \rightarrow 1
$$


and

$$
c(s / r, 1 / r) \rightarrow 1 \quad \text { as } s \rightarrow 1
$$

\section{Main construction}

In this section we state and prove our construction result. Throughout, $D(z, r)$ denotes the open disc with centre $z$ and radius $r$.

THEOREM 3.1. (Main construction) Let $\left(b_{n}\right)_{n \geq 0}$ be a sequence of Blaschke products of corresponding degree $d_{n} \geq 1$, and let $\left(\alpha_{n}\right)_{n \geq 0}$ be a sequence of real numbers with $\alpha_{0}=1$ and $\alpha_{n+1} / \alpha_{n} \leq 1 / 6$. For $n \geq 0$, let

$$
\begin{aligned}
D_{n} & =D\left(9 n, \alpha_{n}\right), \\
\Delta_{n}=D\left(a_{n}, \alpha_{n}\right) \quad \text { and } \quad \Delta_{n}^{\prime} & =D\left(a_{n}, 2 \alpha_{n}\right) \quad \text { where } a_{n}=9 n+4 \alpha_{n},
\end{aligned}
$$

and

$$
G_{n}=D\left(\kappa_{n}, 1\right) \quad \text { and } \quad G_{n}^{\prime}=D\left(\kappa_{n}, 5 / 4\right) \quad \text { where } \kappa_{n}=a_{n}+3 .
$$

We consider the function

$$
\varphi(z)= \begin{cases}z+9 & \text { if } z \in \overline{D_{n}}, n \geq 0, \\ \frac{z-a_{n}}{\alpha_{n}}+\kappa_{n} & \text { if } z \in \overline{\Delta_{n}^{\prime}}, n \geq 0, \\ \alpha_{n+1} b_{n}\left(z-\kappa_{n}\right)+4 \alpha_{n+1} & \text { if } z \in \overline{G_{n}^{\prime}}, n \geq 0,\end{cases}
$$

and define $V_{m}, \zeta_{m}$ and $\rho_{m}$ by

$$
\begin{aligned}
V_{m} & =D\left(\zeta_{m}, \rho_{m}\right) \\
& =\varphi^{m}\left(\Delta_{0}\right)= \begin{cases}\Delta_{n} & \text { if } m=\ell_{n}-1, n \geq 0, \\
G_{n} & \text { if } m=\ell_{n}, n \geq 0, \\
D\left(9 k+4 \alpha_{n+1}, \alpha_{n+1}\right) \subset D_{k} & \text { if } m=\ell_{n}+k+1,0 \leq k \leq n,\end{cases}
\end{aligned}
$$

where $\left(\ell_{n}\right)$ is defined by $\ell_{0}=1$ and $\ell_{n+1}=\ell_{n}+n+3, n \geq 0$.

For a suitable choice of $\left(\alpha_{n}\right)$, there exists a transcendental entire function $f$ having an orbit of bounded, simply connected, oscillating wandering domains $U_{m}$ such that, for $m, n \geq 0$ :

(i) $\overline{D\left(\zeta_{m}, r_{m}\right)} \subset U_{m} \subset D\left(\zeta_{m}, R_{m}\right)$, where $0<r_{m}<\rho_{m}<R_{m}$, and $r_{m} \sim \rho_{m}$ and $R_{m} \sim \rho_{m}$ as $m \rightarrow \infty$;

(ii) $|f(z)-\varphi(z)| \leq \varepsilon_{m}$ on $\overline{D\left(\zeta_{m}, R_{m}\right)}$, where $\varepsilon_{0} \leq 1 / 24$ and $\varepsilon_{\ell_{n}+k}=\alpha_{n+1}^{2} / 2^{k+1}$, for $0 \leq k \leq n+2$;

(iii) $f(9 n)=\varphi(9 n)=9(n+1)$ and $f^{\prime}(9 n)=\varphi^{\prime}(9 n)=1$;

(iv) $f: U_{m} \rightarrow U_{m+1}$ has degree $q_{m}$, where $q_{\ell_{n}}=d_{n}$, and $q_{m}=1$ otherwise. 
Finally, if $z, z^{\prime} \in U_{0}$ and there exists $N \in \mathbb{N}$ such that $f^{\ell_{N}}(z), f^{\ell_{N}}\left(z^{\prime}\right) \in \overline{D\left(\kappa_{N}, r_{\ell_{N}}\right)}$, then, for $n \geq N$, we have

$$
k_{n} \operatorname{dist}_{G_{n}}\left(f^{\ell_{n}}(z), f^{\ell_{n}}\left(z^{\prime}\right)\right) \leq \operatorname{dist}_{U_{\ell_{n}}}\left(f^{\ell_{n}}(z), f^{\ell_{n}}\left(z^{\prime}\right)\right) \leq K_{n} \operatorname{dist}_{G_{n}}\left(f^{\ell_{n}}(z), f^{\ell_{n}}\left(z^{\prime}\right)\right),
$$

where $0<k_{n}<1<K_{n}$ with $k_{n}, K_{n} \rightarrow 1$ as $n \rightarrow \infty$.

Remark 3.2. If the Blaschke products $b_{n}$ are real-symmetric for each $n \geq 0$, then $f$ can be taken to be real-symmetric; see Remark 2.3.

Proof. We consider the sets $V_{m}=\varphi^{m}\left(\Delta_{0}\right)$, where $\Delta_{0}=D(4,1)$, as defined in the statement of Theorem 3.1, and construct a function $f$ which is sufficiently close to $\varphi$ in parts of the plane in order to ensure that $f$ has a bounded wandering domain $U$ with $f^{m}(U)$ close to $V_{m}$, for $m \geq 0$, in the sense that the Hausdorff distance between $U_{m}$ and $V_{m}$ tends to 0 as $m \rightarrow \infty$.

3.1. The sets $V_{m}$. We begin by noting that it follows from the definition of $\varphi$ and the fact that $\alpha_{m+1} / \alpha_{m} \leq 1 / 6$, for $m \geq 0$, that, for each $n \geq 0$,

$$
\begin{gathered}
\varphi\left(\Delta_{n}\right)=G_{n}, \\
\varphi^{2}\left(\Delta_{n}\right)=D\left(4 \alpha_{n+1}, \alpha_{n+1}\right) \subset D\left(0, \alpha_{0}\right)=D_{0},
\end{gathered}
$$

so, for $0 \leq k \leq n$,

$$
\varphi^{k+2}\left(\Delta_{n}\right)=D\left(9 k+4 \alpha_{n+1}, \alpha_{n+1}\right) \subset D\left(9 k, \alpha_{k}\right)=D_{k}
$$

and

$$
\varphi^{n+3}\left(\Delta_{n}\right)=D\left(9(n+1)+4 \alpha_{n+1}, \alpha_{n+1}\right)=\Delta_{n+1} .
$$

This gives the following properties of $V_{m}$, stated in Theorem 3.1:

$$
\begin{aligned}
V_{m} & =D\left(\zeta_{m}, \rho_{m}\right) \\
& =\varphi^{m}\left(\Delta_{0}\right)= \begin{cases}\Delta_{n} & \text { if } m=\ell_{n}-1, n \geq 0, \\
G_{n} & \text { if } m=\ell_{n}, n \geq 0, \\
D\left(9 k+4 \alpha_{n+1}, \alpha_{n+1}\right) \subset D_{k} & \text { if } m=\ell_{n}+k+1,0 \leq k \leq n,\end{cases}
\end{aligned}
$$

where $\left(\ell_{n}\right)$ is defined by $\ell_{0}=1$ and $\ell_{n+1}=\ell_{n}+n+3$, for $n \geq 0$.

In words, if $V_{m} \subset D_{0}$, then $\varphi$ repeatedly translates $V_{m}$ to the right by 9 until the translated image lands on $\Delta_{n}$, for some $n \in \mathbb{N}$, at which point $\varphi$ maps the disc $\Delta_{n}$ onto $G_{n}$ and then maps $G_{n}$ into $D_{0}$ (see Figure 1).

3.2. Construction of the circles $\gamma_{n}$ and $\Gamma_{n}$. We now give an inductive definition of the values $r_{m}$ and $R_{m}$ described in Theorem 3.1(i) and define $\alpha_{n}$ inductively at the same time. We will choose these values in such a way that, if we define

$$
\gamma_{m}=\left\{z:\left|z-\zeta_{m}\right|=r_{m}\right\} \quad \text { and } \quad \Gamma_{m}=\left\{z:\left|z-\zeta_{m}\right|=R_{m}\right\}
$$




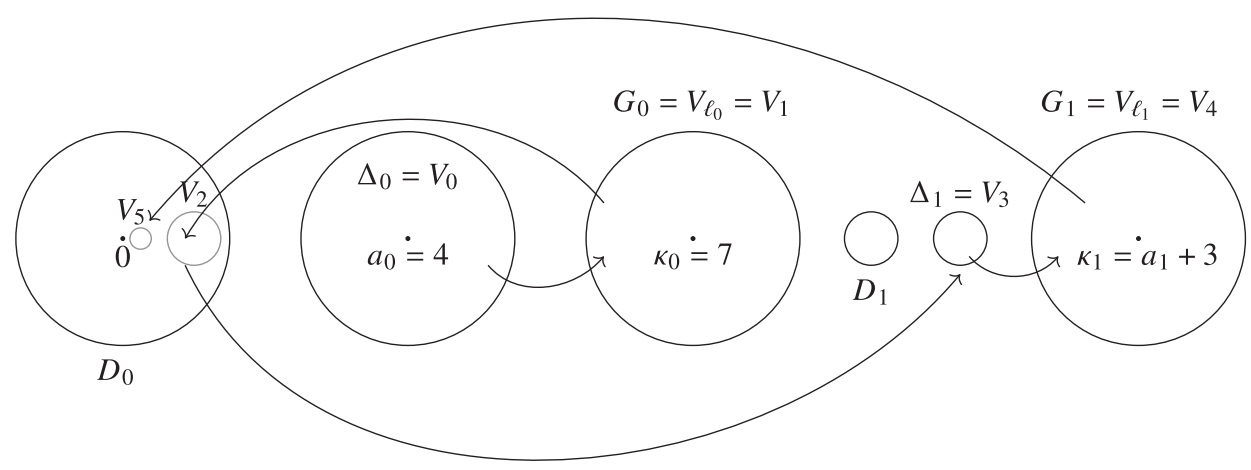

FIGURE 1. The action of the model function $\varphi$.

then, for $m \geq 0$,

$$
\gamma_{m+1} \text { surrounds } \varphi\left(\gamma_{m}\right)
$$

and

$$
\varphi\left(\Gamma_{m}\right) \text { surrounds } \Gamma_{m+1} \text {. }
$$

Further, we choose these values in such a way that we are able to use Lemma 2.2 and Lemma 2.4 to approximate the map $\varphi$ by an entire function $f$ such that $\varphi$ can be replaced by $f$ in (3.3) and (3.4). This in turn allows us to apply Theorem 2.1 to deduce that $f$ has wandering domains with the required properties.

Our construction uses the Blaschke products $b_{n}$ which, for $n \geq 0$, we write as

$$
b_{n}(z)=e^{i \theta_{n}} \prod_{j=1}^{d_{n}} \frac{z+p_{n, j}}{1+\overline{p_{n, j}} z},
$$

where $p_{n, j} \in \mathbb{D}=\{z:|z|<1\}$ are not necessarily different from each other, and $\theta_{n} \in$ $[0,2 \pi)$. We also use the maps defined by

$$
B_{n}(z)=b_{n}\left(z-\kappa_{n}\right) \text { for } n \geq 0,
$$

noting that $B_{n}$ has degree $d_{n}$ and maps $G_{n}$ to $D_{0}=\mathbb{D}$.

First take

$$
r_{0} \in(5 / 6,1) \quad \text { and } \quad R_{0} \in(1,7 / 6),
$$

and recall that $\alpha_{0}=1$. We then choose $r_{1}$ such that

$$
0<1-r_{1} \leq \min \left\{\frac{1-r_{0}}{2}, \operatorname{dist}\left(\varphi\left(\gamma_{0}\right), \partial G_{0}\right)^{2}\right\}
$$

and

$B_{0}\left(\gamma_{1}\right)$ winds exactly $d_{0}$ times round $D(0,1 / 2)$, 
so

$$
\varphi\left(\gamma_{1}\right) \text { winds exactly } d_{0} \text { times round } D\left(\zeta_{2}, \rho_{2} / 2\right),
$$

and choose $R_{1}$ such that

$$
0<R_{1}-1 \leq \min \left\{\frac{R_{0}-1}{2}, \operatorname{dist}\left(\varphi\left(\Gamma_{0}\right), \partial G_{0}\right), \frac{1}{\max _{j}\left|p_{0}, j\right|-1}\right\} .
$$

Now assume that, for some $n \geq 0, \alpha_{k}$ has been chosen for $0 \leq k \leq n$, and $r_{m}$ and $R_{m}$ have been chosen for $0 \leq m \leq \ell_{n}$. (Note that $\ell_{0}=1$ and we have already specified $\alpha_{0}, r_{0}$, $R_{0}, r_{1}$ and $R_{1}$.) We shall give a rule for choosing $\alpha_{n+1}$ and also for choosing $r_{m}$ and $R_{m}$ for $\ell_{n}+1 \leq m \leq \ell_{n+1}$. There are three different cases, depending on the value of $m$.

Case 1. First we consider the case when $m=\ell_{n}+1$ (and so $V_{m} \subset D_{0}$ ). We also specify $\alpha_{n+1}$ as part of this case.

We begin by choosing $c_{n+1}, C_{n+1}$ to be circles centred at 0 , lying in the interior and exterior of $D_{0}$ respectively, such that

$$
\operatorname{dist}\left(c_{n+1}, \partial D_{0}\right) \leq \min \left\{\frac{\rho_{\ell_{n}}-r_{\ell_{n}}}{6}, \frac{1}{2} \operatorname{dist}\left(B_{n}\left(\gamma_{\ell_{n}}\right), \partial D_{0}\right)\right\}
$$

and

$$
\operatorname{dist}\left(C_{n+1}, \partial D_{0}\right) \leq \min \left\{\frac{R_{\ell_{n}}-\rho_{\ell_{n}}}{6}, \operatorname{dist}\left(c_{n+1}, \partial D_{0}\right), \frac{1}{2} \operatorname{dist}\left(B_{n}\left(\Gamma_{\ell_{n}}\right), \partial D_{0}\right)\right\} .
$$

We set

$$
\alpha_{n+1}=\operatorname{dist}\left(C_{n+1}, \partial D_{0}\right)
$$

and note, using the fact that $\varphi(z)=\alpha_{n+1} B_{n}(z)+4 \alpha_{n+1}$, for $z \in G_{n}^{\prime}$, that $V_{\ell_{n}+1}=$ $\varphi\left(V_{\ell_{n}}\right)=D\left(4 \alpha_{n+1}, \alpha_{n+1}\right)$, so $\rho_{\ell_{n}+1}=\alpha_{n+1}$. We then set

$$
r_{\ell_{n}+1}=\rho_{\ell_{n}+1}-\alpha_{n+1}^{2}
$$

and

$$
R_{\ell_{n}+1}=\rho_{\ell_{n}+1}+\alpha_{n+1}^{2} .
$$

Note that, together with (3.9), (3.11) and (3.10), these definitions imply that

$$
\begin{gathered}
\rho_{\ell_{n}+1}-r_{\ell_{n}+1}=\alpha_{n+1}^{2} \leq \alpha_{n+1} \operatorname{dist}\left(c_{n+1}, \partial D_{0}\right) \leq \frac{1}{2} \operatorname{dist}\left(\varphi\left(\gamma \ell_{n}\right), \partial V_{\ell_{n}+1}\right), \\
R_{\ell_{n}+1}-\rho_{\ell_{n}+1}=\alpha_{n+1}^{2}=\alpha_{n+1} \operatorname{dist}\left(C_{n+1}, \partial D_{0}\right) \leq \frac{1}{2} \operatorname{dist}\left(\varphi\left(\Gamma_{\ell_{n}}\right), \partial V_{\ell_{n}+1}\right),
\end{gathered}
$$

and hence

$$
R_{\ell_{n}+1}-r_{\ell_{n}+1}=2 \alpha_{n+1}^{2} \leq \min \left\{\operatorname{dist}\left(\varphi\left(\gamma_{\ell_{n}}\right), \partial V_{\ell_{n}+1}\right), \operatorname{dist}\left(\varphi\left(\Gamma_{\ell_{n}}\right), \partial V_{\ell_{n}+1}\right)\right\} .
$$

Case 2. We now consider the cases when $m=\ell_{n}+k+1$, for $1 \leq k \leq n+1$. Then

$$
V_{\ell_{n}+k+1}=\varphi^{k}\left(V_{\ell_{n}+1}\right) \subset D_{k} \quad \text { for } 1 \leq k \leq n,
$$

and

$$
V_{\ell_{n}+n+2}=V_{\ell_{n+1}-1}=D\left(9(n+1)+4 \alpha_{n+1}, \alpha_{n+1}\right)=\Delta_{n+1} \text {. }
$$


In all these cases, we simply choose $r_{m}$ and $R_{m}$ to satisfy

$$
\rho_{m}-r_{m}=\frac{\rho_{m-1}-r_{m-1}}{2}
$$

and

$$
R_{m}-\rho_{m}=\frac{R_{m-1}-\rho_{m-1}}{2} .
$$

Case 3. Finally, we consider the case when $m=\ell_{n+1}=\ell_{n}+n+3$, so $V_{m}=G_{n+1}$. In this case, we choose $r_{\ell_{n+1}}$ and $R_{\ell_{n+1}}$ so that

$$
\begin{gathered}
0<\rho_{\ell_{n+1}}-r_{\ell_{n+1}} \leq \min \left\{\frac{\rho_{\ell_{n+1}-1}-r_{\ell_{n+1}-1}}{2}, \operatorname{dist}\left(\varphi\left(\gamma_{\ell_{n+1}-1}\right), \partial G_{n+1}\right)^{2}\right\}, \\
B_{n+1}\left(\gamma_{\ell_{n+1}}\right) \text { winds exactly } d_{n+1} \text { times round } D(0,1 / 2), \\
0<R_{\ell_{n+1}}-\rho_{\ell_{n+1}} \\
\leq \min \left\{\frac{R_{\ell_{n+1}-1}-\rho_{\ell_{n+1}-1}}{2}, \operatorname{dist}\left(\varphi\left(\Gamma_{\ell_{n+1}-1}\right), \partial G_{n+1}\right), \frac{1}{\max _{j}\left\{\left|p_{n+1, j}\right|\right\}}-1\right\} .
\end{gathered}
$$

This inductive process defines the values $r_{m}$ and $R_{m}$, and hence the circles $\gamma_{m}$ and $\Gamma_{m}$, for $m \geq 0$. Note that it follows from (3.10), (3.11), (3.18), (3.13) and (3.21) that

$$
\alpha_{n+1} \leq \frac{R_{\ell_{n}}-\rho_{\ell_{n}}}{6}<\frac{R_{\ell_{n-1}+1}-\rho_{\ell_{n-1}+1}}{6}=\frac{\alpha_{n}^{2}}{6}<\frac{\alpha_{n}}{6} \quad \text { for } n \geq 1 .
$$

Moreover, it follows from the definition of $\varphi$ together with (3.5) and (3.2) that we have

$$
\varphi(z)=\alpha_{n+1} B_{n}(z)+4 \alpha_{n+1}=\rho_{\ell_{n}+1} B_{n}(z)+\zeta_{\ell_{n}+1} \quad \text { for } z \in G_{n}, n \geq 0 .
$$

So (3.20) implies that, for $n \geq 0$,

$$
\varphi\left(\gamma_{\ell_{n+1}}\right) \text { winds exactly } d_{n+1} \text { times round } D\left(\zeta_{\ell_{n+1}+1}, \rho_{\ell_{n+1}+1} / 2\right) \text {. }
$$

We also note that it follows from (3.15), (3.18) and (3.2) that, for $m=\ell_{n}+k+1$, where $0 \leq k \leq n+1$, we have $R_{m}-\rho_{m} \leq R_{\ell_{n}+1}-\rho_{\ell_{n}+1}<\alpha_{n+1}=\rho_{m}$. So, for $m=\ell_{n}+k+1$, where $0 \leq k \leq n$, we have

$$
V_{m}^{\prime}=D\left(\zeta_{m}, R_{m}\right) \subset D\left(\zeta_{m}, 2 \rho_{m}\right)=D\left(9 k+4 \alpha_{n+1}, 2 \alpha_{n+1}\right) \subset D\left(9 k, 6 \alpha_{n+1}\right) \subset D_{k}
$$

and

$$
V_{\ell_{n}+n+2}^{\prime}=V_{\ell_{n+1}-1}^{\prime}=D\left(\zeta_{\ell_{n+1}-1}, R_{\ell_{n+1}-1}\right) \subset \Delta_{n+1}^{\prime}
$$

by the definitions of $D\left(\zeta_{m}, \rho_{m}\right)$ and $\Delta_{n}^{\prime}$ in the statement of Theorem 3.1.

It then follows from (3.16), and the fact that $\alpha_{n}<1 / 2$ for $n>0$, that

$$
V_{\ell_{n+1}}^{\prime}=D\left(\zeta_{\ell_{n+1}}, R_{\ell_{n+1}}\right) \subset G_{n+1}^{\prime} .
$$

Also, $\varphi$ is analytic in $V_{\ell_{n+1}}^{\prime}$ by (3.22) together with the last condition in (3.21).

It follows from (3.14) that, for $n \geq 0$,

$$
\gamma_{\ell_{n}+1} \text { surrounds } \varphi\left(\gamma_{\ell_{n}}\right),
$$




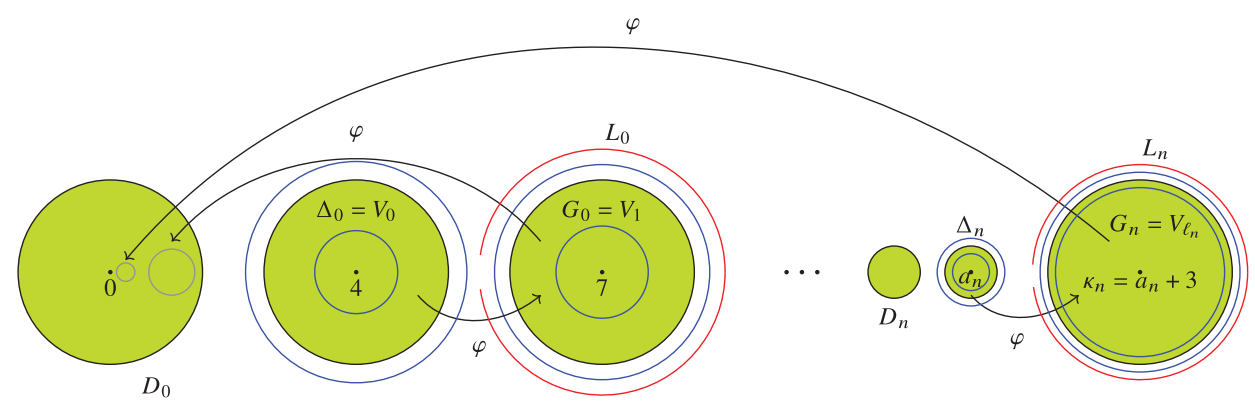

FIGURE 2. Sketch of the set-up of Theorem 3.1, showing the location of the circles $\gamma_{n}$ and $\Gamma_{n}$ (in blue), and the $\operatorname{arcs} L_{n}$ (in red). (Colour available online.)

and from (3.15) that, for $n \geq 0$,

$$
\varphi\left(\Gamma_{\ell_{n}}\right) \text { surrounds } \Gamma_{\ell_{n}+1}
$$

Thus (3.3) and (3.4) hold when $m=\ell_{n}$, where $n \geq 0$.

Also, if $m=\ell_{n}+k+1$, where $n \geq 0,0 \leq k \leq n+1$, then $\varphi$ is a translation on $\gamma_{m}$ and $\Gamma_{m}$, by (3.24) and the definition of $\varphi$. Since, by (3.17), we have

$$
\rho_{m+1}-r_{m+1} \leq \frac{\rho_{m}-r_{m}}{2}
$$

and, by (3.18),

$$
R_{m+1}-\rho_{m+1} \leq \frac{R_{m}-\rho_{m}}{2},
$$

it follows that (3.3) and (3.4) hold for these values of $m$ too.

Finally, it follows from (3.25) that, on $\gamma_{\ell_{n+1}-1}$ and $\Gamma_{\ell_{n+1}-1}, n \geq 0$, the function $\varphi$ is a scaling by a factor of $1 / \alpha_{n+1}>1$ followed by a translation, and so it follows from (3.19) and (3.21) that (3.3) and (3.4) hold in this case too.

We note that the sets $\overline{V_{m}^{\prime}}$ are disjoint since, if $V_{\ell_{n}+k+1}^{\prime}, V_{\ell_{n+1}+k+1}^{\prime} \subset D_{k}$, for some $n \geq 0,0 \leq k \leq n$, then $V_{\ell_{n}+k+1}^{\prime} \subset D\left(9 k+4 \alpha_{n+1}, 2 \alpha_{n+1}\right)$ and $V_{\ell_{n+1}+k+1}^{\prime} \subset D(9 k+$ $\left.4 \alpha_{n+2}, 2 \alpha_{n+2}\right)$, and

$$
D\left(9 k+4 \alpha_{n+1}, 2 \alpha_{n+1}\right) \cap D\left(9 k+4 \alpha_{n+2}, 2 \alpha_{n+2}\right)=\emptyset,
$$

since

$$
4 \alpha_{n+2}+2 \alpha_{n+2}=6 \alpha_{n+2} \leq \alpha_{n+1}<4 \alpha_{n+1}-2 \alpha_{n+1}
$$

3.3. Construction of the function $f$. Our aim now is to use Lemma 2.2 and Lemma 2.4 to approximate the map $\varphi$ by a single entire function $f$ such that, for $m \geq 0, \gamma_{m+1}$ surrounds $f\left(\gamma_{m}\right)$ and $f\left(\Gamma_{m}\right)$ surrounds $\Gamma_{m+1}$. We also require $f$ to map certain curves $L_{n}$ near $\overline{G_{n}}$ in such a way that we can apply Theorem 2.1.

We define $L_{n}$, for $n \geq 0$, to be the circular arc

$$
L_{n}:=\left\{z:\left|z-a_{n}\right|=R_{\ell_{n}}+\delta_{\ell_{n}}^{2} / 2,\left|\arg \left(z-a_{n}\right)\right| \leq \pi-\delta_{\ell_{n}}^{2}\right\},
$$

where $\delta_{m}=R_{m}-r_{m} \rightarrow 0$ as $m \rightarrow \infty$; see Figure 2 . 
We also define approximation error quantities $\varepsilon_{m}$, for $m \geq 0$, by

$$
\varepsilon_{m}=\min \left\{\frac{1}{4} \operatorname{dist}\left(\varphi\left(\gamma_{m}\right), \partial V_{m+1}\right), \frac{1}{4} \operatorname{dist}\left(\varphi\left(\Gamma_{m}\right), \partial V_{m+1}\right), \frac{1}{4} \delta_{m+1}\right\}>0 .
$$

We now show that these errors have the upper bounds stated in part (ii) of our theorem. First, it follows from (3.6), (3.7) and (3.8) that

$$
\varepsilon_{0} \leq\left(R_{1}-r_{1}\right) / 4 \leq\left(R_{0}-r_{0}\right) / 8<1 / 24 .
$$

Next we note that it follows from (3.16) that, for $n \geq 0$,

$$
\varepsilon_{\ell_{n}}=\delta_{\ell_{n}+1} / 4=\left(R_{\ell_{n}+1}-r_{\ell_{n}+1}\right) / 4=2 \alpha_{n+1}^{2} / 4=\alpha_{n+1}^{2} / 2 .
$$

It then follows from (3.17), (3.18), (3.19) and (3.21) that, for $0 \leq k \leq n+1, n \geq 0$,

$$
\varepsilon_{\ell_{n}+k+1}=\left(R_{\ell_{n}+k+2}-r_{\ell_{n}+k+2}\right) / 4=\left(R_{\ell_{n}+1}-r_{\ell_{n}+1}\right) / 2^{k+3}=\alpha_{n+1}^{2} / 2^{k+2} .
$$

Thus

$$
\varepsilon_{\ell_{n}+k}=\alpha_{n+1}^{2} / 2^{k+1} \quad \text { for } 0 \leq k \leq n+2, n \geq 0,
$$

as required for part (ii).

Since $\varphi$ is analytic in each set $\overline{V_{\ell_{n+1}}^{\prime}}$, for $n \geq 0$, it follows from Lemma 2.2 that there exists an entire function $f$ such that, for $n \geq 0$,

$$
\begin{gathered}
|f(z)-\varphi(z)|<\varepsilon_{\ell_{n}+n+1} \quad \text { for } z \in \overline{D_{n}}, \\
|f(z)-\varphi(z)|<\varepsilon_{\ell_{n}-1} \quad \text { for } z \in \overline{\Delta_{n}^{\prime}}, \\
|f(z)-\varphi(z)|<\varepsilon_{\ell_{n}} \quad \text { for } z \in \overline{G_{n}^{\prime}}, \\
f(9 n)=9(n+1), \\
f^{\prime}(9 n)=1,
\end{gathered}
$$

and such that

$$
|f(z)+4| \leq 1 / 2 \quad \text { for } z \in \overline{D(-4,1)} \cup \bigcup_{n \geq 0} L_{n} .
$$

It follows from (3.33), (3.36) and (3.37) that for each $k \geq 0$ we can apply Lemma 2.4 in the disc $D_{k}=D\left(9 k, \alpha_{k}\right)$, with $g(z)=f(z)-\varphi(z), R=\alpha_{k}$ and associated constant $\epsilon=\varepsilon_{\ell_{k}+k+1} / \alpha_{k}$. Note that the conditions of Lemma 2.4 are satisfied since it follows from (3.32) that

$$
\epsilon=\varepsilon_{\ell_{k}+k+1} / \alpha_{k}=\frac{\alpha_{k+1}^{2}}{\alpha_{k} 2^{k+2}}<\frac{\alpha_{k+1}}{6}<1 / 4 \text { for } k \geq 0 .
$$

So, by Lemma 2.4, for all $z \in D_{k}, k \geq 0$, we have

$$
|f(z)-\varphi(z)| \leq \frac{\varepsilon \ell_{k}+k+1}{\alpha_{k}^{2}}|z-9 k|^{2} .
$$

We will now show that this implies that, for each $m \geq 0$,

$$
|f(z)-\varphi(z)|<\varepsilon_{m} \quad \text { for } z \in \overline{V_{m}^{\prime}} .
$$


First we note that (3.40) follows from (3.25) and (3.26) together with (3.34) and (3.35) when $m=\ell_{n}$ or $m=\ell_{n}-1$, for some $n \geq 0$. Other values of $m$ are of the form $m=$ $\ell_{n}+k+1$, for some $n \geq 0,0 \leq k \leq n$, and it follows from (3.24) that, in this case,

$$
V_{m}^{\prime} \subset D\left(9 k, 6 \alpha_{n+1}\right) \subset D_{k} .
$$

Therefore, by (3.39), (3.32) and using the fact that $\alpha_{k+1} \leq \alpha_{k} / 6$, we have, for $z \in$ $V_{\ell_{n}+k+1}, n \geq 0$ and $0 \leq k \leq n$,

$$
\begin{aligned}
|f(z)-\varphi(z)| & \leq \frac{\varepsilon_{\ell_{k}+k+1}}{\alpha_{k}^{2}}\left(6 \alpha_{n+1}\right)^{2} \\
& =\frac{\alpha_{k+1}^{2}}{2^{k+2} \alpha_{k}^{2}} 36 \alpha_{n+1}^{2} \\
& \leq \frac{\alpha_{n+1}^{2}}{2^{k+2}}=\varepsilon_{\ell_{n}+k+1} .
\end{aligned}
$$

Thus (3.40) holds for all $m \geq 0$.

It now follows from (3.3), (3.4), (3.30) and (3.40) that, for $m \geq 0$,

$$
\begin{aligned}
& \gamma_{m+1} \text { surrounds } f\left(\gamma_{m}\right), \\
& f\left(\Gamma_{m}\right) \text { surrounds } \Gamma_{m+1} .
\end{aligned}
$$

We now apply Theorem 2.1 to the Jordan curves $\gamma_{m}, \Gamma_{m}, m \geq 0$, the compact curves $L_{n}$, $n \geq 0$, and the bounded domain $D=D(-4,1)$, noting that these sets satisfy the required hypotheses by construction and by (3.41), (3.42), (3.29) and (3.38). Part (i) of Theorem 3.1 now follows from Theorem 2.1, part (ii) follows from (3.40) together with the upper bounds for the errors that we obtained earlier, and part (iii) follows from (3.36) and (3.37).

Next we outline the proof of part (iv). The fact that $f: U_{\ell_{n+1}} \rightarrow U_{\ell_{n+1}+1}$ has degree $d_{n+1}$ follows from the final statement of Theorem 2.1, since (3.23), (3.30) and (3.35) together imply that $f\left(\gamma_{\ell_{n+1}}\right)$ and $f\left(\Gamma_{\ell_{n+1}}\right)$ both wind exactly $d_{n+1}$ times round the disc $D\left(\zeta_{\ell_{n+1}+1}, \rho_{\ell_{n+1}+1} / 2\right)$; for the details of this argument see the proof of [BEF+21, Theorem 5.3]. Since $\varphi$ is univalent in all other cases, the same argument applies to show that $f: U_{m} \rightarrow U_{m+1}$ is univalent in all other cases.

To complete the proof of Theorem 3.1, we note that the double inequality that compares the hyperbolic distances in $U_{\ell_{n}}$ between points of two orbits under $f$ with the corresponding hyperbolic distances in the discs $G_{n}$ follows by applying Lemma 2.5 with

$$
s=1-\frac{3}{4} \operatorname{dist}\left(\varphi\left(\gamma_{\ell_{n}-1}\right), \partial G_{n}\right), \quad r=r_{\ell_{n}} \quad \text { and } \quad R=R_{\ell_{n}},
$$

and noting that $f^{\ell_{n+1}-\ell_{n}}\left(\overline{D\left(\kappa_{n}, r_{\ell_{n}}\right)} \subset D\left(\kappa_{n+1}, r_{\ell_{n+1}}\right)\right.$; we omit the details, which are similar to those given in the proof of the final statement of [BEF+21, Theorem 5.3].

\section{Preliminary results for Theorem 1.1}

In this section we prove some results which we use in order to construct our examples. In particular, we obtain estimates on the orbits of points in a wandering domain $U$ of a transcendental entire function $f$ obtained by applying Theorem 3.1 with specific Blaschke 
products $b_{n}$. Our first result is used repeatedly in our constructions and gives estimates of the distances between orbits under the function $f$ and under the model function $\varphi$.

LEMMA 4.1. Let f be a transcendental entire function with a wandering domain $U$ arising from applying Theorem 3.1 with the Blaschke products $b_{n}$. Then, using the notation of Theorem 3.1,

(a) if $z, z^{\prime} \in U_{\ell_{n}}$, for some $n \geq 0$, we have

$$
\left|f^{n+3}(z)-\varphi^{n+3}\left(z^{\prime}\right)\right| \leq \alpha_{n+1}+\left|b_{n}\left(z-\kappa_{n}\right)-b_{n}\left(z^{\prime}-\kappa_{n}\right)\right|
$$

(b) and hence, if $z, z^{\prime} \in \overline{D\left(\zeta_{0}, r_{0}\right)} \subset U_{0}$, we have

$$
\left|f^{\ell_{n+1}}(z)-\varphi^{\ell_{n+1}}\left(z^{\prime}\right)\right| \leq \alpha_{n+1}+\left|b_{n}\left(f^{\ell_{n}}(z)-\kappa_{n}\right)-b_{n}\left(\varphi^{\ell_{n}}\left(z^{\prime}\right)-\kappa_{n}\right)\right| .
$$

Proof. To prove part (a), we begin by considering the case that $z=z^{\prime}$. We first use induction to show that, if $z \in U_{\ell_{n}}$, for some $n \geq 0$, then

$$
\left|f^{m}(z)-\varphi^{m}(z)\right| \leq \sum_{k=0}^{m-1} \varepsilon_{\ell_{n}+k} \text { for } 1 \leq m \leq n+2 .
$$

We note that, for $m=1$, this holds by Theorem 3.1(ii). Now assume that (4.1) holds for some $m, 1 \leq m<n+2$. We have

$$
\left|f^{m+1}(z)-\varphi^{m+1}(z)\right| \leq\left|f\left(f^{m}(z)\right)-\varphi\left(f^{m}(z)\right)\right|+\left|\varphi\left(f^{m}(z)\right)-\varphi^{m+1}(z)\right| .
$$

Since $z \in U_{\ell_{n}}$, we have $f^{m}(z) \in U_{\ell_{n}+m} \subset D\left(\zeta_{\ell_{n}+m}, R_{\ell_{n}+m}\right)$ and $\varphi^{m}(z) \in D\left(\zeta_{\ell_{n}+m}\right.$, $\left.R_{\ell_{n}+m}\right)$. Also, $\varphi$ is a translation on $D\left(\zeta_{\ell_{n}+m}, R_{\ell_{n}+m}\right)$ and so, together with Theorem 3.1(ii), we can deduce from (4.2) that

$$
\left|f^{m+1}(z)-\varphi^{m+1}(z)\right| \leq \varepsilon_{\ell_{n}+m}+\left|f^{m}(z)-\varphi^{m}(z)\right| \leq \sum_{k=0}^{m} \varepsilon_{\ell_{n}+k} .
$$

Thus (4.1) holds as claimed.

Next, we note that for $z \in U_{\ell_{n}}$ we have $f^{n+2}(z), \varphi^{n+2}(z) \in \Delta_{n}^{\prime}$, on which $\varphi$ is a scaling by a factor of $1 / \alpha_{n+1}$ followed by a translation and so, by (4.1) and Theorem 3.1(ii),

$$
\begin{aligned}
\left|f^{n+3}(z)-\varphi^{n+3}(z)\right| & \leq\left|f\left(f^{n+2}(z)\right)-\varphi\left(f^{n+2}(z)\right)\right|+\left|\varphi\left(f^{n+2}(z)\right)-\varphi^{n+3}(z)\right| \\
& \leq \varepsilon \ell_{n}+n+2+\frac{1}{\alpha_{n+1}} \sum_{k=0}^{n+1} \varepsilon \ell_{n}+k \\
& \leq \frac{\alpha_{n+1}}{2^{n+3}}+\alpha_{n+1} \sum_{k=0}^{n+1} \frac{1}{2^{k+1}} \\
& <\alpha_{n+1} .
\end{aligned}
$$

This shows that

$$
\left|f^{n+3}(z)-\varphi^{n+3}(z)\right| \leq \alpha_{n+1},
$$

which is the result of part (a) in the case that $z=z^{\prime}$. 
We now use this fact to prove part (a) in general. If $z, z^{\prime} \in U_{\ell_{n}}$, for some $n \geq 0$, then it follows from (4.3) and the definition of $\varphi$ that

$$
\begin{aligned}
\left|f^{n+3}(z)-\varphi^{n+3}\left(z^{\prime}\right)\right| & \leq\left|f^{n+3}(z)-\varphi^{n+3}(z)\right|+\left|\varphi^{n+3}(z)-\varphi^{n+3}\left(z^{\prime}\right)\right| \\
& \leq \alpha_{n+1}+\left|b_{n}\left(z-\kappa_{n}\right)-b_{n}\left(z^{\prime}-\kappa_{n}\right)\right| .
\end{aligned}
$$

This completes the proof of part (a).

Now we suppose that $z, z^{\prime} \in \overline{D\left(\zeta_{0}, r_{0}\right)}$. It follows from Theorem 3.1(i) that $z \in U_{0}$ and hence $f^{\ell_{n}}(z) \in U_{\ell_{n}}$, for $n \geq 0$. It also follows from (3.3) in the proof of Theorem 3.1 that $\varphi^{\ell_{n}}\left(z^{\prime}\right) \in D\left(\zeta_{\ell_{n}}, r_{\ell_{n}}\right)$ and hence, by Theorem 3.1(i), that $\varphi^{\ell_{n}}\left(z^{\prime}\right) \in U_{\ell_{n}}$, for $n \geq 0$. So part (b) follows from part (a) by replacing $z$ and $z^{\prime}$ by $f^{\ell_{n}}(z)$ and $\varphi^{\ell_{n}}\left(z^{\prime}\right)$, respectively.

Our next result gives a precise estimate for a Blaschke product that is used in one of our examples. We use this result in the proof of Lemma 4.3(a).

LEMMA 4.2. Let $b(z)=((z+1 / 3) /(1+z / 3))^{2}$ and suppose that $0<r<1$. Then $0<$ $r<b(r)<1$ and

$$
\left|\frac{b(x)-b(r)}{x-r}\right|<\frac{b^{2}(r)-b(r)}{b(r)-r} \text { for } 0<x<b(r) .
$$

Proof. Our proof is based on a useful relationship between the cross-ratio of four points $a<b<c<d$ on $\mathbb{R}$, defined as

$$
(a, b, c, d)=\frac{(b-a)(d-c)}{(d-a)(c-b)},
$$

and the Schwarzian derivative of a real function $f$, defined as

$$
S f=\frac{f^{\prime \prime \prime}}{f^{\prime \prime}}-\frac{3}{2}\left(\frac{f^{\prime \prime}}{f^{\prime}}\right)^{2}
$$

It is well known that if $f$ is monotonic on an interval $I$ and $S f<0$ on $I$, then

$$
(f(a), f(b), f(c), f(d))<(a, b, c, d) \quad \text { whenever } a, b, c, d \in I, a<b<c<d .
$$

See, for example, de Melo and van Strien [dMvS89, §1] for a good account of the relationship between functions with negative Schwarzian and the cross-ratio, including a proof of the above fact. Other key properties (also mentioned in [dMvS89]) are that Möbius maps have zero Schwarzian and the composition rule for Schwarzians is

$$
S(g \circ f)(x)=S g(f(x))\left(f^{\prime}(x)\right)^{2}+S f(x) .
$$

Since the Schwarzian derivative of a Möbius map is zero on its domain in $\mathbb{R}$, it follows immediately from this composition rule that the function $b$ has negative Schwarzian on the interval $(-3, \infty)$.

It is straightforward to check that 1 is a fixed point of the function $b$ and that $r<b(r)<$ 1 , for $r \in(0,1)$. Note also that $b$ is increasing on $(-1 / 3, \infty)$ and convex on $(-3,1)$. We first prove (4.4) in the case when $r<x<b(r)$, by considering the four points $r, x, b(r), 1$. 
Since $b$ is increasing on $(-1 / 3, \infty)$ and has negative Schwarzian there, we deduce that

$$
\frac{(b(x)-b(r))\left(1-b^{2}(r)\right)}{(1-b(r))\left(b^{2}(r)-b(x)\right)}<\frac{(x-r)(1-b(r))}{(1-r)(b(r)-x)} .
$$

Since $b$ is convex on $(0,1)$ we deduce that

$$
\frac{1-b(r)}{1-r}<\frac{1-b^{2}(r)}{1-b(r)} .
$$

We deduce from the previous two inequalities that

$$
\frac{b(x)-b(r)}{b^{2}(r)-b(x)}<\frac{(1-b(r))^{2}}{(1-r)\left(1-b^{2}(r)\right)} \frac{x-r}{b(r)-x}<\frac{x-r}{b(r)-x},
$$

and hence (by taking reciprocals and adding 1 to both sides) that

$$
\frac{b(x)-b(r)}{b^{2}(r)-b(r)}<\frac{x-r}{b(r)-r} .
$$

This proves (4.4) in the case when $r<x<b(r)$.

For the case when $0<x<r$, similar reasoning can be used with the points $x, r, b(r), 1$, to deduce that

$$
\frac{b(r)-b(x)}{b^{2}(r)-b(r)}<\frac{r-x}{b(r)-r} .
$$

This completes the proof of Lemma 4.2.

The following lemma describes dynamical properties of the transcendental entire functions arising from Theorem 3.1 when using specific Blaschke products of a certain form. Two of our examples will be constructed using these Blaschke products. The proof of this result takes several pages.

LEMMA 4.3. Let $b$ be a Blaschke product of degree 2, and let $f$ be an entire function arising by applying Theorem 3.1 with $b_{n}=b$, for $n \geq 0$.

(a) If $b(z)=((z+1 / 3) /(1+z / 3))^{2}$ then there exist $x, y \in U_{0} \cap \mathbb{R}, N \in \mathbb{N}$ and $c>0$, with $f^{n}(x) \neq f^{n}(y)$ for $n \geq 0$, such that

$$
f^{\ell_{N}}(x)=\kappa_{N} \quad \text { and } \quad \kappa_{n}+1-f^{\ell_{n}}(x) \sim \frac{c}{n^{1 / 2}} \quad \text { as } n \rightarrow \infty
$$

and

$$
f^{\ell_{N}}(y)=\kappa_{N}+1 / 9 \quad \text { and } \quad \kappa_{n}+1-f^{\ell_{n}}(y) \sim \frac{c}{(n+1)^{1 / 2}} \quad \text { as } n \rightarrow \infty,
$$

and moreover,

$$
f^{\ell_{n}}(y)-f^{\ell_{n}}(x)=\frac{O(1)}{n^{3 / 2}} \quad \text { as } n \rightarrow \infty .
$$

(b) If $b(z)=((z+1 / 2) /(1+z / 2))^{2}$ then there exist $x, y \in U_{0} \cap \mathbb{R}$ and $N \in \mathbb{N}$, with $f^{n}(x) \neq f^{n}(y)$ for $n \geq 0$, such that

$$
f^{\ell_{N}}(x)=\kappa_{n} \quad \text { and } \quad \kappa_{n}+1-f^{\ell_{n}}(x)=c \lambda^{n}\left(1+\eta_{n}\right) \quad \text { for } n \geq N,
$$


and

$$
f^{\ell_{N}}(y)=\kappa_{n}+1 / 4 \quad \text { and } \quad \kappa_{n}+1-f^{\ell_{n}}(y)=c \lambda^{n+1}\left(1+\xi_{n}\right) \quad \text { for } n \geq N,
$$

where $c>0, \lambda=2 / 3$ and $\max \left\{\left|\eta_{n}\right|,\left|\xi_{n}\right|\right\} \leq 1 / 10$, for $n \geq N$.

Proof. First we observe that in both parts it is sufficient to prove the stated results about the behaviours of $f^{\ell_{n}-\ell_{N}}\left(x_{N}\right)$ and $f^{\ell_{n}-\ell_{N}}\left(y_{N}\right)$ when $x_{N}, y_{N} \in U_{\ell_{N}}$ for some particular positive integer $N$.

(a) Recall that, by the analysis of the behaviour in $\mathbb{D}$ of the iterates of $b$ near its parabolic fixed point 1 (see [BEF+21, Lemma 6.2(c)], for example), there are positive constants $c$ and $d$ such that

$$
1-b^{n}(0) \sim \frac{c}{n^{1 / 2}} \quad \text { as } n \rightarrow \infty
$$

and

$$
b^{n+1}(0)-b^{n}(0) \sim \frac{d}{n^{3 / 2}} \quad \text { as } n \rightarrow \infty .
$$

Therefore, we can choose $N$ so large that

$$
\frac{d}{2 n^{3 / 2}}<b^{n+1}(0)-b^{n}(0)<\frac{2 d}{n^{3 / 2}} \text { for } n \geq N,
$$

and also such that

$$
\frac{4}{d 6^{n}} \leq \frac{1}{10} \text { for } n \geq N
$$

We then take $r_{n}=b^{n-N}(0)$, for $n \geq N$, and define

$$
x_{N}=\kappa_{N} \quad \text { and } \quad x_{n+1}=f^{n+3}\left(x_{n}\right) \text { for } n \geq N,
$$

and

$$
x_{n}^{\prime}:=\kappa_{n}+r_{n} \in G_{n} \cap \mathbb{R} \text { for } n \geq N .
$$

It follows from the definition of $\varphi$ that

$$
x_{n+1}^{\prime}=\varphi^{n+3}\left(x_{n}^{\prime}\right)=b\left(x_{n}^{\prime}-\kappa_{n}\right) \quad \text { for } n \geq N .
$$

We use Lemma 4.2 to show that the orbit of $x_{N}=\kappa_{N}$ under $f$ closely follows that of $x_{N}$ under $\varphi$. More precisely, we shall show that

$$
\left|x_{n}-x_{n}^{\prime}\right|<\frac{1}{10}\left(r_{n+1}-r_{n}\right) \quad \text { for } n \geq N+1 .
$$

Note that it follows from (4.10) that $x_{n}^{\prime} \in U_{\ell_{n}} \cap \mathbb{R}$ since $x_{N}^{\prime}=x_{N} \in U_{\ell_{N}} \cap \mathbb{R}$ and $f$ is a real entire function.

We shall prove (4.11) by using induction to show that

$$
\left|x_{n}-x_{n}^{\prime}\right| \leq\left(r_{n+1}-r_{n}\right) \sum_{k=N+1}^{n} \frac{1}{6^{k}\left(r_{k+1}-r_{k}\right)} \quad \text { for } n \geq N+1 .
$$


Before proving (4.12), we show that it implies (4.11). Using (4.8) and (4.9), it follows that, for $n \geq N+1$,

$$
\begin{aligned}
\frac{\left|x_{n}-x_{n}^{\prime}\right|}{r_{n+1}-r_{n}} & \leq \sum_{k=N+1}^{n} \frac{1}{6^{k}\left(r_{k+1}-r_{k}\right)} \\
& \leq \sum_{k=N+1}^{n} \frac{2(k-N)^{3 / 2}}{d 6^{k}} \\
& =\frac{2}{d 6^{N+1}} \sum_{j=0}^{n-N-1} \frac{(j+1)^{3 / 2}}{6^{j}} \\
& \leq \frac{4}{d 6^{N+1}}<\frac{1}{10},
\end{aligned}
$$

since the sum in the penultimate expression is dominated by the geometric series $1+$ $1 / 2+1 / 4+\cdots$. Thus (4.11) holds.

To start the proof of (4.12), we have

$$
\left|x_{N+1}-x_{N+1}^{\prime}\right|=\left|f^{N+3}\left(x_{N}\right)-\varphi^{N+3}\left(x_{N}\right)\right| \leq \frac{1}{6^{N+1}},
$$

by Theorem 3.1(iv), since $x_{N} \in U_{\ell_{N}}$. Now we assume that (4.12) holds for some $n \geq N+$ 1 and deduce that it holds for $n+1$. Note that, whenever (4.11) holds (and so whenever (4.12) holds), we have $x_{n}^{\prime} \in\left[\kappa_{n}, \kappa_{n}+1\right)$, by the definition of $x_{n}$.

By the definition of $\varphi$, Lemma 4.1(a), Lemma 4.2 and Theorem 3.1(iv), we have

$$
\begin{aligned}
\left|x_{n+1}-x_{n+1}^{\prime}\right| & \leq \alpha_{n+1}+\left|b\left(x_{n}-\kappa_{n}\right)-b\left(x_{n}^{\prime}-\kappa_{n}\right)\right| \\
& \leq \frac{1}{6^{n+1}}+\left|x_{n}-x_{n}^{\prime}\right|\left(\frac{b^{2}\left(x_{n}^{\prime}-\kappa_{n}\right)-b\left(x_{n}^{\prime}-\kappa_{n}\right)}{b\left(x_{n}^{\prime}-\kappa_{n}\right)-\left(x_{n}^{\prime}-\kappa_{n}\right)}\right) \\
& =\frac{1}{6^{n+1}}+\left|x_{n}-x_{n}^{\prime}\right|\left(\frac{r_{n+2}-r_{n+1}}{r_{n+1}-r_{n}}\right) \\
& \leq \frac{1}{6^{n+1}}+\left(r_{n+2}-r_{n+1}\right) \sum_{k=N+1}^{n} \frac{1}{6^{k}\left(r_{k+1}-r_{k}\right)} \\
& =\left(r_{n+2}-r_{n+1}\right) \sum_{k=N+1}^{n+1} \frac{1}{6^{k}\left(r_{k+1}-r_{k}\right)} .
\end{aligned}
$$

This proves (4.12), so (4.11) holds.

Next, we define

$$
y_{N}=\kappa_{N}+b(0)=\kappa_{N}+1 / 9 \text { and } y_{n+1}=f^{n+3}\left(y_{n}\right) \text { for } n \geq N,
$$

and

$$
y_{n}^{\prime}:=\kappa_{n}+r_{n+1} \in G_{n} \cap \mathbb{R} \text { for } n \geq N,
$$


with the same value of $N$ as used earlier. Then

$$
y_{n}^{\prime}-x_{n}^{\prime}=r_{n+1}-r_{n}=b^{n+1-N}(0)-b^{n-N}(0) \quad \text { for } n \geq N,
$$

and

$$
y_{n+1}^{\prime}=\varphi^{n+3}\left(y_{n}^{\prime}\right)=b\left(y_{n}^{\prime}-\kappa_{n}\right) \quad \text { for } n \geq N .
$$

Reasoning as above, we obtain

$$
\left|y_{n}-y_{n}^{\prime}\right| \leq \frac{1}{10}\left(r_{n+2}-r_{n+1}\right) \text { for } n \geq N+1 .
$$

Combining (4.6) and (4.7) with (4.11) and (4.14), we obtain

$$
\kappa_{n}+1-x_{n} \sim \frac{c}{n^{1 / 2}} \quad \text { as } n \rightarrow \infty
$$

and

$$
\begin{aligned}
\left|y_{n}-x_{n}\right| & \leq\left|y_{n}-y_{n}^{\prime}\right|+\left|y_{n}^{\prime}-x_{n}^{\prime}\right|+\left|x_{n}^{\prime}-x_{n}\right| \\
& \leq \frac{1}{10}\left(r_{n+2}-r_{n+1}\right)+\left(r_{n+1}-r_{n}\right)+\frac{1}{10}\left(r_{n+1}-r_{n}\right) \\
& =\frac{O(1)}{n^{3 / 2}} \quad \text { as } n \rightarrow \infty,
\end{aligned}
$$

which gives the required result by taking $x, y \in U_{0}$ such that $f^{\ell_{N}}(x)=x_{N}=\kappa_{N}$ and $f^{\ell_{N}}(y)=y_{N}=\kappa_{N}+b(0)=\kappa_{N}+1 / 9$. Note that $y_{n} \neq x_{n}$ for $n \geq N$, by (4.11), (4.13) and (4.14), so we deduce that $f^{n}(x) \neq f^{n}(y)$ for $n \geq 0$.

(b) The proof of part (b) is similar to that of part (a), and we outline the argument briefly.

As in part (a), we take $r_{n}=b^{n-N}(0)$, for $n \geq N$, for some sufficiently large $N \in \mathbb{N}$ to be specified later in the proof, and put

$$
x_{N}=\kappa_{N} \quad \text { and } \quad x_{n+1}=f^{n+3}\left(x_{n}\right) \text { for } n \geq N,
$$

and

$$
x_{n}^{\prime}:=\kappa_{n}+r_{n} \in G_{n} \cap \mathbb{R} \text { for } n \geq N,
$$

so once again

$$
x_{n+1}^{\prime}=\varphi^{n+3}\left(x_{n}^{\prime}\right)=b\left(x_{n}^{\prime}-\kappa_{n}\right) \quad \text { for } n \geq N .
$$

Now note that the function $b$ has fixed point 1 with multiplier $\lambda=2 / 3$. It follows that

$$
1-r_{n+1}=\lambda\left(1-r_{n}\right)\left(1+O\left(1-r_{n}\right)\right) \text { as } n \rightarrow \infty,
$$

so, for some constant $c>0$,

$$
\kappa_{n}+1-x_{n}=1-r_{n} \sim c \lambda^{n} \quad \text { as } n \rightarrow \infty .
$$

Also, since $b$ is univalent in the disc $\{z:|z-1|<1\}$ (or by a direct calculation), we have

$$
\left|b^{\prime}(z)\right| \leq \lambda(1+C|z-1|) \quad \text { for }|z-1|<1 / 2,
$$


where $C$ is a positive constant, and so

$$
|b(w)-b(z)| \leq \lambda(1+C|z-1|)|w-z| \text { for }|w-z|<1 / 4,|z-1|<1 / 4 .
$$

As in part (a), we show that the orbit of $x_{N}$ under $f$ closely follows that of $x_{N}$ under $\varphi$. To be precise, we claim that for $N$ sufficiently large we have

$$
\left|x_{n}-x_{n}^{\prime}\right| \leq \frac{c}{10} \lambda^{n} \quad \text { for } n \geq N .
$$

Indeed, for $n \geq N$, we have

$$
\begin{aligned}
\left|x_{n+1}-x_{n+1}^{\prime}\right| & \leq \alpha_{n+1}+\left|b\left(x_{n}-\kappa_{n}\right)-b\left(x_{n}-\kappa_{n}\right)\right| \\
& \leq \frac{1}{6^{n+1}}+\lambda\left(1+C\left(1-r_{n}\right)\right)\left|x_{n}-x_{n}^{\prime}\right|,
\end{aligned}
$$

by Lemma 4.1(a), Theorem 3.1(iv), (4.17) and (4.18), provided that $N$ is sufficiently large. Since $x_{N}=x_{N}^{\prime}=\kappa_{N}$, it follows easily by induction that, for $n \geq N+1$, we have

$$
\delta_{n} \leq\left(\prod_{k=N+1}^{\infty}\left(1+C\left(1-r_{k}\right)\right)\right)\left(\sum_{k=N+1}^{n} \frac{1}{(6 \lambda)^{k}}\right) \quad \text { where } \delta_{n}=\frac{\left|x_{n}-x_{n}^{\prime}\right|}{\lambda^{n}},
$$

and (4.19) easily follows by (4.17) and by taking $N$ sufficiently large.

We obtain the first estimate in part (b) by taking $x \in U_{0}$ such that $f^{\ell_{N}}(x)=x_{N}=\kappa_{N}$. The second estimate follows by a similar argument, but this time we use an orbit under $f$ whose subsequence passing through $U_{\ell_{n}}, n \geq N$, closely follows the sequence $y_{n}^{\prime}:=$ $\kappa_{n}+r_{n+1}, n \geq N$, by taking $y \in U_{0}$ such that $f^{\ell_{N}}(y)=y_{N}=\kappa_{N}+b(0)=\kappa_{N}+1 / 4$. The proof that $f^{n}(x) \neq f^{n}(y)$ for $n \geq 0$ uses (4.19) and is similar to that in part (a).

Finally in this section, we give several estimates for a Blaschke product used in another of our examples.

LEMMA 4.4. For $n \geq 0$, let $b_{n}(z)=\widetilde{\mu_{n}}\left(\mu_{n}(z)^{2}\right)$, where

$$
\mu_{n}(z)=\frac{z+s_{n}}{1+s_{n} z} \quad \text { and } \quad \widetilde{\mu_{n}}(z)=\frac{z-s_{n}^{2}}{1-s_{n}^{2} z},
$$

and let

$$
\lambda_{n}=\frac{2 s_{n}}{1+s_{n}^{2}}
$$

where $s_{n} \in(0,1)$. Then, for $n \geq 0$,

$$
\lambda_{n} x \leq\left(\frac{x+\lambda_{n}}{1+\lambda_{n} x}\right) x=b_{n}(x) \leq x \quad \text { for } 0<x<1,
$$

and

$$
\lambda_{n}(y-x) \leq b_{n}(y)-b_{n}(x) \leq \frac{2}{1+\lambda_{n}}(y-x) \quad \text { for } 0 \leq x<y<1 .
$$


Proof. For $x \in(0,1)$ and $n \geq 0$, we have

$$
\begin{aligned}
b_{n}(x) & =\frac{\left(\left(x+s_{n}\right) /\left(1+s_{n} x\right)\right)^{2}-s_{n}^{2}}{1-s_{n}^{2}\left(\left(x+s_{n}\right) /\left(1+s_{n} x\right)\right)^{2}} \\
& =\frac{\left(x+s_{n}\right)^{2}-s_{n}^{2}\left(1+s_{n} x\right)^{2}}{\left(1+s_{n} x\right)^{2}-s_{n}^{2}\left(x+s_{n}\right)^{2}} \\
& =\frac{x^{2}+2 s_{n} x-2 s_{n}^{3} x-s_{n}^{4} x^{2}}{1+2 s_{n} x-2 s_{n}^{3} x-s_{n}^{4}} \\
& =\left(\frac{\left(1-s_{n}^{4}\right) x+\left(1-s_{n}^{2}\right) 2 s_{n}}{1-s_{n}^{4}+\left(1-s_{n}^{2}\right) 2 s_{n} x}\right) x \\
& =\left(\frac{\left(1+s_{n}^{2}\right) x+2 s_{n}}{1+s_{n}^{2}+2 s_{n} x}\right) x \\
& =\left(\frac{x+\lambda_{n}}{1+\lambda_{n} x}\right) x .
\end{aligned}
$$

Since

$$
a \leq \frac{x+a}{1+a x} \leq 1
$$

for $x, a \in[0,1]$, part (a) follows.

For part (b), we deduce from the expression for $b_{n}$ given in part (a) that, for $0 \leq x<$ $y<1$ and $n \geq 0$,

$$
\begin{aligned}
b_{n}(y)-b_{n}(x) & =y\left(\frac{y+\lambda_{n}}{1+\lambda_{n} y}\right)-x\left(\frac{x+\lambda_{n}}{1+\lambda_{n} x}\right) \\
& =(y-x) \frac{y+x+\lambda_{n}(1+x y)}{\left(1+\lambda_{n} y\right)\left(1+\lambda_{n} x\right)},
\end{aligned}
$$

and the conclusion then easily follows from the facts that $0<\lambda_{n}<1$, for $n \geq 0$, and $0 \leq$ $x<y<1$.

\section{Proof of Theorem 1.1.}

In this section we construct six examples of bounded oscillating wandering domains, based on the two simply connected wandering domain classifications given in [BEF+21]. First, in terms of hyperbolic distances between orbits of points, simply connected wandering domains are classified as follows $[\mathbf{B E F}+\mathbf{2 1}$, Theorem A].

THEOREM 5.1. (First classification theorem) Let $U$ be a simply connected wandering domain of a transcendental entire function $f$ and let $U_{n}$ be the Fatou component containing $f^{n}(U)$, for $n \in \mathbb{N}$. Define the countable set of pairs

$$
E=\left\{\left(z, z^{\prime}\right) \in U \times U: f^{k}(z)=f^{k}\left(z^{\prime}\right) \text { for some } k \in \mathbb{N}\right\} .
$$

Then exactly one of the following statements holds.

(1) $\operatorname{dist}_{U_{n}}\left(f^{n}(z), f^{n}\left(z^{\prime}\right)\right) \underset{n \rightarrow \infty}{\longrightarrow} c\left(z, z^{\prime}\right)=0$ for all $z, z^{\prime} \in U$, and we say that $U$ is (hyperbolically) contracting. 
(2) $\operatorname{dist}_{U_{n}}\left(f^{n}(z), f^{n}\left(z^{\prime}\right)\right) \underset{n \rightarrow \infty}{\longrightarrow} c\left(z, z^{\prime}\right)>0$ and $\operatorname{dist}_{U_{n}}\left(f^{n}(z), f^{n}\left(z^{\prime}\right)\right) \neq c\left(z, z^{\prime}\right)$ for all $\left(z, z^{\prime}\right) \in(U \times U) \backslash E, n \in \mathbb{N}$, and we say that $U$ is (hyperbolically) semicontracting.

(3) There exists $N>0$ such that for all $n \geq N$, $\operatorname{dist}_{U_{n}}\left(f^{n}(z), f^{n}\left(z^{\prime}\right)\right)=c\left(z, z^{\prime}\right)>0$ for all $\left(z, z^{\prime}\right) \in(U \times U) \backslash E$, and we say that $U$ is (hyperbolically) eventually isometric.

Next, in terms of convergence of orbits to the boundary there are again three types of simply connected wandering domains (see $[\mathrm{BEF}+21$, Theorem $\mathrm{C}]$ ), though only the latter two types ((b) and (c)) are realizable for oscillating wandering domains as explained in the introduction.

THEOREM 5.2. (Second classification theorem) Let $U$ be a simply connected wandering domain of a transcendental entire function $f$ and let $U_{n}$ be the Fatou component containing $f^{n}(U)$, for $n \in \mathbb{N}$. Then exactly one of the following statements holds.

(a) $\lim _{\inf _{n \rightarrow \infty}} \operatorname{dist}\left(f^{n}(z), \partial U_{n}\right)>0$ for all $z \in U$, that is, all orbits stay away from the boundary.

(b) There exists a subsequence $n_{k} \rightarrow \infty$ for which $\operatorname{dist}\left(f^{n_{k}}(z), \partial U_{n_{k}}\right) \rightarrow 0$ for all $z \in$ $U$, while for a different subsequence $m_{k} \rightarrow \infty$ we have that

$$
\liminf _{k \rightarrow \infty} \operatorname{dist}\left(f^{m_{k}}(z), \partial U_{m_{k}}\right)>0 \quad \text { for } z \in U .
$$

(c) $\operatorname{dist}\left(f^{n}(z), \partial U_{n}\right) \rightarrow 0$ for all $z \in U$, that is, all orbits converge to the boundary.

Each of the examples in this section is constructed by applying Theorem 3.1 with an appropriate choice of the Blaschke products $b_{n}$. We make repeated use of the following two results.

LEMMA 5.3. Let f be a transcendental entire function with an orbit of wandering domains $\left(U_{n}\right)$ arising from applying Theorem 3.1 with the Blaschke products $\left(b_{n}\right)_{n \geq 0}$ and suppose that there exist $s, t \in U_{0}, N \in \mathbb{N}$ with

$$
f^{\ell_{N}}(s), f^{\ell_{N}}(t) \in \overline{D\left(\kappa_{N}, r_{\ell_{N}}\right)},
$$

where the sequences $\left(\ell_{n}\right),\left(\kappa_{n}\right)$ and $\left(r_{n}\right)$ are as defined in Theorem 3.1.

(a) If $\operatorname{dist}_{G_{n}}\left(f^{\ell_{n}}(s), f^{\ell_{n}}(t)\right) \underset{n \rightarrow \infty}{\longrightarrow} 0$ and $f^{\ell_{n}}(s) \neq f^{\ell_{n}}(t)$, for $n \geq 0$, then $U_{0}$ is contracting.

(b) If $\lim \inf _{n \rightarrow \infty} \operatorname{dist}_{G_{n}}\left(f^{\ell_{n}}(s), f^{\ell_{n}}(t)\right)>0$ and $f: U_{n} \rightarrow U_{n+1}$ has degree greater than 1 for infinitely many $n \in \mathbb{N}$, then $U_{0}$ is semi-contracting.

Proof. (a) In this case it follows from the last part of Theorem 3.1 that

$$
\operatorname{dist}_{U_{n}}\left(f^{\ell_{n}}(s), f^{\ell_{n}}(t)\right) \underset{n \rightarrow \infty}{\longrightarrow} 0 .
$$

It now follows from Theorem 5.1 that the only possibility is for $U_{0}$ to be contracting.

(b) In this case it follows from the last part of Theorem 3.1 that

$$
\liminf _{n \rightarrow \infty} \operatorname{dist}_{U_{n}}\left(f^{\ell_{n}}(s), f^{\ell_{n}}(t)\right)>0
$$


and so $U_{0}$ is not contracting. Since $f: U_{n} \rightarrow U_{n+1}$ has degree greater than 1 for infinitely many $n \in \mathbb{N}$, we know that $U_{0}$ is not eventually isometric, and so it follows from Theorem 5.1 that $U_{0}$ is semi-contracting.

LEMMA 5.4. Let f be a transcendental entire function with an orbit of wandering domains $\left(U_{n}\right)$ arising from applying Theorem 3.1 with the Blaschke products $\left(b_{n}\right)_{n \geq 0}$ and let $s \in U_{0}$ with

$$
f^{\ell_{n}}(s) \in G_{n} \quad \text { for } n \geq 0
$$

where the sets $G_{n}$ and the sequence $\left(\ell_{n}\right), n \geq 0$ are as defined in Theorem 3.1.

(a) If $\liminf _{n \rightarrow \infty} \operatorname{dist}\left(f^{\ell_{n}}(s), \partial G_{n}\right)>0$, then orbits of points in $U_{0}$ behave as described in Theorem 5.2(b).

(b) If $\operatorname{dist}\left(f^{\ell_{n}}(s), \partial G_{n}\right) \rightarrow 0$ as $n \rightarrow \infty$, then orbits of points in $U_{0}$ behave as described in Theorem 5.2(c).

Proof. We begin by noting that it follows from Theorem 3.1 that

$$
\begin{aligned}
\varphi^{m}\left(\Delta_{0}\right) & =D\left(\zeta_{m}, \rho_{m}\right) \\
& = \begin{cases}\Delta_{n}=D\left(a_{n}, \alpha_{n}\right) & \text { if } m=\ell_{n}-1 \text { where } n \geq 0, \\
G_{n}=D\left(\kappa_{n}, 1\right) & \text { if } m=\ell_{n}, \text { where } n \geq 0, \\
D\left(9 k+4 \alpha_{n+1}, \alpha_{n+1}\right) \subset D_{k} & \text { if } m=\ell_{n}+k+1, \text { where } 0 \leq k \leq n .\end{cases}
\end{aligned}
$$

Since we know from Theorem 3.1(i) that the wandering domains $U_{m}$ are approximated increasingly well by the sets $\varphi_{m}\left(\Delta_{0}\right)$ as $m \rightarrow \infty$, it follows that $\operatorname{diam} U_{m} \rightarrow 0$ as $m \rightarrow \infty$ for $m \neq \ell_{n}, n \geq 0$. So, if $s \in U_{0}$, then

$$
\operatorname{dist}\left(f^{m}(s), \partial U_{m}\right) \rightarrow 0 \quad \text { as } m \rightarrow \infty, m \neq \ell_{n}, n \geq 0 .
$$

(a) In this case it follows from (5.1) together with Theorem 3.1(i) that

$$
\liminf _{n \rightarrow \infty} \operatorname{dist}\left(f^{\ell_{n}}(s), \partial U_{\ell_{n}}\right)>0
$$

Together with (5.2), this implies that orbits of points in $U_{0}$ behave as described in Theorem 5.2(b).

(b) In this case it follows from (5.1) together with Theorem 3.1(i) that

$$
\operatorname{dist}\left(f^{\ell_{n}}(s), \partial U_{\ell_{n}}\right) \rightarrow 0 \quad \text { as } n \rightarrow \infty .
$$

Together with (5.2), this implies that orbits of points in $U_{0}$ behave as described in Theorem 5.2(c).

In some of the examples we make use of the following estimate for the hyperbolic distance in the unit disc. 
Observation 1. For two points $r, s \in(0,1)$ with $r<s$ we have that

$$
\operatorname{dist}_{\mathbb{D}}(r, s)=\int_{r}^{s} \frac{2 d t}{1-t^{2}},
$$

and so

$$
\log \frac{1-r}{1-s}=\int_{r}^{s} \frac{d t}{1-t} \leq \operatorname{dist}_{\mathbb{D}}(r, s)=\int_{r}^{s} \frac{2 d t}{1-t}=2 \log \frac{1-r}{1-s} .
$$

We now give the examples that together prove Theorem 1.1. Examples 1, 2 and 3, which follow, correspond to the three cases of Theorem 5.1. Within each example we give two functions, corresponding to the two realizable cases of Theorem 5.2.

Example 1. (Two contracting wandering domains) For each of the cases (b) and (c) of Theorem 5.2, there exists a transcendental entire function $f$ having a sequence of bounded, simply connected, oscillating contracting wandering domains $\left(U_{n}\right)$ with the stated behaviour.

Proof. First example. We construct an oscillating contracting wandering domain $U_{0}$ with the behaviour described in Theorem 5.2 (b) by applying Theorem 3.1 with $b_{n}(z)=z^{2}$, for $n \geq 0$.

We begin by considering the orbits of points in the disc $D(4,1 / 12) \subset D\left(4, r_{0}\right)$ under iteration by $\varphi$. We note that, if $z \in D\left(\kappa_{n}, R_{\ell_{n}}\right)$, for some $n \geq 0$, then

$$
\left|\varphi^{n+3}(z)-\kappa_{n+1}\right|=\left|b_{n}\left(z-\kappa_{n}\right)\right|=\left|z-\kappa_{n}\right|^{2} .
$$

So, if $z \in D(4,1 / 12)$, then, for $n \geq 0$, we have

$$
\left|\varphi^{\ell_{n}}(z)-\kappa_{n}\right|=\left|\varphi(z)-\kappa_{0}\right|^{2^{n}}=|z-7|^{2^{n}} \leq(1 / 12)^{2^{n}} \rightarrow 0 \quad \text { as } n \rightarrow \infty .
$$

Next we claim that, if $z \in D(4,1 / 12)$, then

$$
\left|f^{\ell_{n}}(z)-\varphi^{\ell_{n}}(z)\right| \leq \sum_{i=1}^{n} \frac{\alpha_{i}}{3^{n-i}}+\frac{|f(z)-\varphi(z)|}{3^{n}} \quad \text { for } n \geq 0,
$$

and

$$
\left|f^{\ell_{n}}(z)-\kappa_{n}\right| \leq \frac{1}{4} \quad \text { for } n \geq 0 .
$$

We prove (5.6) and (5.5) together using induction. First, we note that they are true when $n=0$, since if $z \in D(4,1 / 12)$, then $\left|f^{\ell_{0}}(z)-\varphi^{\ell_{0}}(z)\right|=|f(z)-\varphi(z)| \leq \varepsilon_{0} \leq 1 / 24$, by Theorem 3.1(ii), and $\left|\varphi^{\ell_{0}}(z)-\kappa_{0}\right|=\left|\varphi(z)-\kappa_{0}\right| \leq 1 / 12$ by (5.4).

Next, we suppose that (5.5) and (5.6) hold for $n=m \geq 0$. It follows from these two estimates and Lemma 4.1(b), together with (5.4), that if $z \in D(4,1 / 12)$, then

$$
\begin{aligned}
\left|f^{\ell_{m+1}}(z)-\varphi^{\ell_{m+1}}(z)\right| & \leq \alpha_{m+1}+\left|b_{m}\left(f^{\ell_{m}}(z)-\kappa_{m}\right)-b_{m}\left(\varphi^{\ell_{m}}(z)-\kappa_{m}\right)\right| \\
& =\alpha_{m+1}+\left|\left(f^{\ell_{m}}(z)-\kappa_{m}\right)^{2}-\left(\varphi^{\ell_{m}}(z)-\kappa_{m}\right)^{2}\right| \\
& \leq \alpha_{m+1}+\left|f^{\ell_{m}}(z)-\varphi^{\ell_{m}}(z)\right|\left(\left|f^{\ell_{m}}(z)-\kappa_{m}\right|+\left|\varphi^{\ell_{m}}(z)-\kappa_{m}\right|\right) \\
& \leq \alpha_{m+1}+\frac{1}{3}\left|f^{\ell_{m}}(z)-\varphi^{\ell_{m}}(z)\right|
\end{aligned}
$$




$$
\begin{aligned}
& \leq \alpha_{m+1}+\frac{1}{3}\left(\sum_{i=1}^{m} \frac{\alpha_{i}}{3^{m-i}}+\frac{|f(z)-\varphi(z)|}{3^{m}}\right) \\
& =\sum_{i=1}^{m+1} \frac{\alpha_{i}}{3^{m+1-i}}+\frac{|f(z)-\varphi(z)|}{3^{m+1}},
\end{aligned}
$$

which gives (5.5) with $n=m+1$, and also

$$
\begin{aligned}
\left|f^{\ell_{m+1}}(z)-\kappa_{m+1}\right| & \leq\left|f^{\ell_{m+1}}(z)-\varphi^{\ell_{m+1}}(z)\right|+\left|\varphi^{\ell_{m+1}}(z)-\kappa_{m+1}\right| \\
& \leq \sum_{i=1}^{m+1} \frac{\alpha_{i}}{3^{m+1-i}}+\frac{|f(z)-\varphi(z)|}{3^{m+1}}+\left(\frac{1}{12}\right)^{2^{m+1}} \\
& \leq \sum_{i=1}^{n} \frac{1}{3^{m+1-i} 6^{i}}+\frac{1}{12} \frac{1}{3^{m+1}}+\left(\frac{1}{12}\right)^{2^{m+1}} \\
& \leq \frac{1}{6}++\frac{1}{12} \frac{1}{3^{m+1}}+\left(\frac{1}{12}\right)^{2^{m+1}}<\frac{1}{4},
\end{aligned}
$$

which gives (5.6) with $n=m+1$.

Since $\alpha_{n} \leq 1 / 6^{n}$ for $n \geq 0$, it follows from (5.5) that if $z \in D(4,1 / 12)$, then

$$
\left|f^{\ell_{n}}(z)-\varphi^{\ell_{n}}(z)\right| \rightarrow 0 \quad \text { as } n \rightarrow \infty .
$$

Together with (5.4), this implies that, if $z \in D(4,1 / 12)$, then

$$
\left|f^{\ell_{n}}(z)-\kappa_{n}\right| \rightarrow 0 \quad \text { as } n \rightarrow \infty \text {. }
$$

We now use (5.7) together with Lemma 5.3 and Lemma 5.4 to show that the wandering domain $U_{0}$ has the required properties. First we take $s, t \in D(4,1 / 12)$ such that $f(s), f(t) \in D\left(\kappa_{0}, r_{1}\right)$ with $f^{\ell_{n}}(s) \neq f^{\ell_{n}}(t)$, for $n \geq 0$. Since $G_{n}=D\left(\kappa_{n}, 1\right)$, for $n \geq 0$, it follows from (5.7) that

$$
\operatorname{dist}_{G_{n}}\left(f^{\ell_{n}}(s), f^{\ell_{n}}(t)\right) \rightarrow 0 \quad \text { as } n \rightarrow \infty
$$

and hence, by Lemma 5.3(a), $U_{0}$ is contracting.

Also, it follows from (5.7) that

$$
\lim _{n \rightarrow \infty} \operatorname{dist}\left(f^{\ell_{n}}(s), \partial G_{n}\right)=1
$$

and hence, by Lemma 5.4(a), orbits of points in $U_{0}$ behave as described in Theorem 5.2(b). Second example. We construct an oscillating contracting wandering domain $U_{0}$ with the behaviour described in Theorem 5.2 (c) by applying Theorem 3.1 with $b_{n}(z)=$ $((z+1 / 3) /(1+z / 3))^{2}$, for $n \geq 0$. Let $x, y \in U_{0}$ be as in Lemma 4.3(a). Since $G_{n}=D\left(\kappa_{n}, 1\right)$, for $n \geq 0$, it follows from Lemma 4.3(a) and the hyperbolic metric 
estimate (5.3) that

$$
\begin{aligned}
\operatorname{dist}_{G_{n}}\left(f^{\ell_{n}}(x), f^{\ell_{n}}(y)\right) & \leq 2 \log \frac{\kappa_{n}+1-f^{\ell_{n}}(x)}{\kappa_{n}+1-f^{\ell_{n}}(y)} \\
& =2 \log \left(1+\frac{f^{\ell_{n}}(t)-f^{\ell_{n}}(x)}{\kappa_{n}+1-f^{\ell_{n}}(y)}\right) \\
& \sim 2 \log \left(1+\frac{O(1) / n^{3 / 2}}{c(n+1)^{1 / 2}}\right) \\
& =\frac{O(1)}{n} \text { as } n \rightarrow \infty .
\end{aligned}
$$

It now follows from Lemma 5.3(a) that $U_{0}$ is contracting. We also know from Lemma 4.3(a) that

$$
\operatorname{dist}\left(f^{\ell_{n}}(x), \partial G_{n}\right) \rightarrow 0 \quad \text { as } n \rightarrow \infty
$$

and so it follows from Lemma 5.4(b) that orbits of points in $U_{0}$ behave as described in Theorem 5.2(c).

Example 2. (Two semi-contracting wandering domains) For each of the cases (b) and (c) of Theorem 5.2, there exists a transcendental entire function $f$ having a sequence of bounded, simply connected, oscillating semi-contracting wandering domains $\left(U_{n}\right)$ with the stated behaviour.

Proof. First example. We construct an oscillating semi-contracting wandering domain with the behaviour described in Theorem 5.2(b) by applying Theorem 3.1 with $b_{n}(z)=$ $\widetilde{\mu_{n}}\left(\mu_{n}(z)^{2}\right)$, for $n \geq 0$, where

$$
\mu_{n}(z)=\frac{z+s_{n}}{1+s_{n} z} \quad \text { and } \quad \widetilde{\mu_{n}}(z)=\frac{z-s_{n}^{2}}{1-s_{n}^{2} z} .
$$

We shall use the estimates for $b_{n}$ obtained in Lemma 4.4, and once again put

$$
\lambda_{n}=\frac{2 s_{n}}{1+s_{n}^{2}} \quad \text { for } n \geq 0 .
$$

We now choose $s_{n} \in(0,1)$ with $s_{n} \rightarrow 1$ as $n \rightarrow \infty$ so quickly that

$$
\prod_{j=0}^{\infty} \lambda_{j} \geq 8 / 9 \text { and } \prod_{j=0}^{\infty} \frac{2}{1+\lambda_{j}} \leq 4 / 3 \text {. }
$$

We first consider the orbit of the point 4 under iteration by $f$, noting that

$$
\varphi^{\ell_{n}}(4)=\kappa_{n} \quad \text { for } n \geq 0 .
$$

It follows from (5.9), Lemma 4.1(b) and (4.20) in Lemma 4.4 that, for $n \geq 0$,

$$
\begin{aligned}
\left|f^{\ell_{n+1}}(4)-\kappa_{n+1}\right| & =\left|f^{\ell_{n+1}}(4)-\varphi^{\ell_{n+1}}(4)\right| \\
& \leq \alpha_{n+1}+\left|b_{n}\left(f^{\ell_{n}}(4)-\kappa_{n}\right)-b_{n}\left(\varphi^{\ell_{n}}(4)-\kappa_{n}\right)\right|
\end{aligned}
$$




$$
\begin{aligned}
& =\alpha_{n+1}+\left|b_{n}\left(f^{\ell_{n}}(4)-\kappa_{n}\right)\right| \\
& \leq \alpha_{n+1}+\left|f^{\ell_{n}}(4)-\kappa_{n}\right| .
\end{aligned}
$$

Together with Theorem 3.1(ii) and (5.9), this implies that, for $n \geq 0$,

$$
\left.\mid f^{\ell_{n}}(4)-\kappa_{n}\right)|\leq| f(4)-\kappa_{0}\left|+\sum_{i=1}^{n-1} \alpha_{i}=\right| f(4)-\varphi(4) \mid+\sum_{i=1}^{n-1} \alpha_{i} \leq \frac{1}{24}+\sum_{i=1}^{n-1} \frac{1}{6^{i}}<\frac{1}{4} \text {. }
$$

Now we consider the orbit of $19 / 4$ under $f$. Once again, we begin by considering the orbit under $\varphi$. We claim that

$$
\varphi^{\ell_{n}}(19 / 4)-\kappa_{n} \geq \frac{3}{4} \prod_{i=0}^{n-1} \lambda_{i}
$$

We prove (5.11) by induction, first noting that it holds for $n=0$, since $\varphi^{\ell_{0}}(19 / 4)=$ $\varphi(19 / 4)=\kappa_{0}+3 / 4$. Next, suppose that (5.11) holds for $n=m$. Then, by (4.20),

$$
\begin{aligned}
\varphi^{\ell_{m+1}}(19 / 4)-\kappa_{m+1} & =b_{n}\left(\varphi^{\ell_{m}}(19 / 4)-\kappa_{m}\right) \\
& \geq \lambda_{m}\left(\varphi^{\ell_{m}}(19 / 4)-\kappa_{m}\right) \\
& \geq \frac{3}{4} \lambda_{m} \prod_{i=0}^{m-1} \lambda_{i}=\frac{3}{4} \prod_{i=0}^{m} \lambda_{i} .
\end{aligned}
$$

Thus (5.11) holds for $n=m+1$ and hence, by induction, for all $n \geq 0$. Together with (5.8), this implies that

$$
\varphi^{\ell_{n}}(19 / 4)-\kappa_{n} \geq \frac{2}{3} \quad \text { for } n \geq 0 .
$$

Next we claim that, for $n \geq 0$,

$$
\left|f^{\ell_{n}}(19 / 4)-\varphi^{\ell_{n}}(19 / 4)\right| \leq \sum_{i=1}^{n} \alpha_{i} \prod_{j=i}^{n-1} \frac{2}{1+\lambda_{j}}+\prod_{i=0}^{n-1} \frac{2}{1+\lambda_{i}}|f(19 / 4)-\varphi(19 / 4)| \text {. }
$$

We prove (5.13) by induction, noting that it holds for $n=0$. Next, suppose that (5.13) holds for $n=m \geq 0$. Then it follows from Lemma 4.1(b) and (4.21) that

$$
\begin{aligned}
& \left|f^{\ell_{m+1}}(19 / 4)-\varphi^{\ell_{m+1}}(19 / 4)\right| \\
& \quad \leq \alpha_{m+1}+\left|b_{m}\left(f^{\ell_{m}}(19 / 4)-\kappa_{m}\right)-b_{m}\left(\varphi^{\ell_{n}}(19 / 4)-\kappa_{m}\right)\right| \\
& \quad \leq \alpha_{m+1}+\frac{2}{1+\lambda_{m}}\left|f^{\ell_{m}}(19 / 4)-\varphi^{\ell_{m}}(19 / 4)\right| \\
& \quad \leq \alpha_{m+1}+\frac{2}{1+\lambda_{m}}\left(\sum_{i=1}^{m} \alpha_{i} \prod_{j=i}^{m-1} \frac{2}{1+\lambda_{j}}+\prod_{i=0}^{m-1} \frac{2}{1+\lambda_{i}}|f(19 / 4)-\varphi(19 / 4)|\right) \\
& \quad=\sum_{i=1}^{m+1} \alpha_{i} \prod_{j=i}^{m} \frac{2}{1+\lambda_{j}}+\prod_{i=0}^{m} \frac{2}{1+\lambda_{i}}|f(19 / 4)-\varphi(19 / 4)| .
\end{aligned}
$$


Thus (5.13) holds for $n=m+1$ and hence, by induction, for all $n \geq 0$. It now follows from Theorem 3.1(ii) and (5.8) that

$$
\left|f^{\ell_{n}}(19 / 4)-\varphi^{\ell_{n}}(19 / 4)\right| \leq \prod_{j=0}^{\infty} \frac{2}{1+\lambda_{j}}\left(\sum_{i=1}^{\infty} \frac{1}{6^{i}}+\frac{1}{24}\right) \leq \frac{1}{4} \prod_{j=0}^{\infty} \frac{2}{1+\lambda_{j}} \leq \frac{1}{3} .
$$

It follows from (5.12), (5.14) and (5.10) that, for $n \geq 0$,

$$
\begin{aligned}
\left|f^{\ell_{n}}(19 / 4)-f^{\ell_{n}}(4)\right| & =\left|\varphi^{\ell_{n}}(19 / 4)-\kappa_{n}+f^{\ell_{n}}(19 / 4)-\varphi^{\ell_{n}}(19 / 4)+\kappa_{n}-f^{\ell_{n}}(4)\right| \\
& \geq\left|\varphi^{\ell_{n}}(19 / 4)-\kappa_{n}\right|-\left|f^{\ell_{n}}(19 / 4)-\varphi^{\ell_{n}}(19 / 4)\right|-\left|f^{\ell_{n}}(4)-\kappa_{n}\right| \\
& \geq \frac{2}{3}-\frac{1}{3}-\frac{1}{4}=\frac{1}{12} .
\end{aligned}
$$

Since $G_{n}=D\left(\kappa_{n}, 1\right)$, for $n \geq 0$, together with (5.10) this implies that

$$
\liminf _{n \rightarrow \infty} \operatorname{dist}_{G_{n}}\left(f^{\ell_{n}}(19 / 4), f^{\ell_{n}}(4)\right)>0 .
$$

Also, it follows from Theorem 3.1(iv) that $f: U_{\ell_{n}} \rightarrow U_{\ell_{n+1}}$ has degree greater than 1, for $n \geq 0$. So, by Lemma 5.3(b), $U_{0}$ is semi-contracting.

Finally, it follows from (5.10) that

$$
\liminf _{n \rightarrow \infty} \operatorname{dist}\left(f^{\ell_{n}}(4), \partial G_{n}\right)>0
$$

and so, by Lemma 5.4(a), orbits of points in $U_{0}$ behave as described in Theorem 5.2(b).

Second example. We construct an oscillating semi-contracting wandering domain $U_{0}$ with the behaviour described in Theorem 5.2(c) by applying Theorem 3.1 with $b_{n}(z)=$ $((z+1 / 2) /(1+z / 2))^{2}$, for $n \geq 0$. Let $x, y \in U$ be as in Lemma 4.3(b). Since $G_{n}=$ $D\left(\kappa_{n}, 1\right)$, for $n \geq 0$, it follows from Lemma 4.3(b) and the hyperbolic metric estimate (5.3) that for $n$ sufficiently large we have

$$
\begin{aligned}
\operatorname{dist}_{G_{n}}\left(f^{\ell_{n}}(x), f^{\ell_{n}}(y)\right) & \geq \log \frac{\kappa_{n}+1-f^{\ell_{n}}(x)}{\kappa_{n}+1-f^{\ell_{n}}(y)} \\
& \geq \log \frac{c \lambda^{n}(1-1 / 10)}{c \lambda^{n+1}(1+1 / 10)} \\
& =\log \frac{9}{11 \lambda}=\log \frac{27}{22}>0,
\end{aligned}
$$

recalling that $\lambda=2 / 3$.

Also, it follows from Theorem 3.1 that $f: U_{\ell_{n}} \rightarrow U_{\ell_{n}+1}$ has degree greater than 1 , for $n \geq 0$, so $U_{0}$ is semi-contracting by Lemma 5.3(b). Finally, we know from Lemma 4.3(b) that

$$
\operatorname{dist}\left(f^{\ell_{n}}(x), \partial G_{n}\right) \rightarrow 0 \quad \text { as } n \rightarrow \infty
$$

and so, by Lemma 5.4(b), orbits of points in $U_{0}$ behave as described in Theorem 5.2(c).

Example 3. (Two eventually isometric wandering domains) For each of the cases (b) and (c) of Theorem 5.2, there exists a transcendental entire function $f$ having a sequence of 
bounded, simply connected, oscillating eventually isometric wandering domains $\left(U_{n}\right)$ with the stated behaviour.

Proof. First example. We construct an oscillating eventually isometric wandering domain $U_{0}$ with the behaviour described in Theorem 5.2(b) by applying Theorem 3.1 with $b_{n}(z)=z$, for $n \geq 0$.

Since $b_{n}$ is univalent, for $n \geq 0$, it follows from Theorem 3.1(iv) that $f: U_{m} \rightarrow U_{m+1}$ is also univalent, for $m \geq 0$. Thus $U$ is eventually isometric.

We now consider the orbit of 4 under iteration by $f$. We claim that, for $n \geq 0$,

$$
\left|f^{\ell_{n}}(4)-\varphi^{\ell_{n}}(4)\right| \leq \sum_{i=1}^{n} \alpha_{i}+|f(4)-\varphi(4)| .
$$

We prove (5.15) by induction, noting that it is true for $n=0$. Next, suppose that (5.15) holds for $n=m \geq 0$. Then it follows from Lemma 4.1(b) that

$$
\begin{aligned}
\left|f^{\ell_{m+1}}(4)-\varphi^{\ell_{m+1}}(4)\right| & =\alpha_{m+1}+\left|b_{m}\left(f^{\ell_{m}}(4)-\kappa_{m}\right)-b_{m}\left(\varphi^{\ell_{m}}(4)-\kappa_{m}\right)\right| \\
& =\alpha_{m+1}+\left|f^{\ell_{m}}(4)-\varphi^{\ell_{m}}(4)\right| \\
& =\alpha_{m+1}+\sum_{i=1}^{m} \alpha_{i}+|f(4)-\varphi(4)| \\
& =\sum_{i=1}^{m+1} \alpha_{i}+|f(4)-\varphi(4)| .
\end{aligned}
$$

Thus (5.15) holds for $n=m+1$ and hence, by induction, for all $n \geq 0$.

Since $\alpha_{n} \leq 1 / 6^{n}$ for $n \geq 0$, and $|f(4)-\varphi(4)| \leq 1 / 24$, by Theorem 3.1(ii), it follows from (5.15) that

$$
\left|f^{\ell_{n}}(4)-\varphi^{\ell_{n}}(4)\right| \leq 1 / 2 \text { for } n \geq 0 .
$$

Since $\varphi^{\ell_{n}}(4)=\kappa_{n}$ and $G_{n}=D\left(\kappa_{n}, 1\right)$, for $n \geq 0$, it follows from Lemma 5.4(a) that orbits of points in $U_{0}$ behave as described in Theorem 5.2(b).

Second example. We construct an oscillating eventually isometric wandering domain $U_{0}$ with the behaviour described in Theorem 5.2(c) by applying Theorem 3.1 with $b_{n}(z)=$ $b(z)=(z+5 / 6) /(1+5 z / 6)$, for $n \geq 0$.

Since $b_{n}$ is univalent, for $n \geq 0$, it follows from Theorem 3.1(iv) that $f: U_{m} \rightarrow U_{m+1}$ is also univalent, for $m \geq 0$. Thus $U_{0}$ is eventually isometric.

We now consider the orbit of 4 under iteration by $\varphi$, noting that

$$
\varphi^{\ell_{n}}(4)=\kappa_{n}+b^{n}(0) \quad \text { for } n \geq 0 .
$$

The Blaschke product $b$ has an attracting fixed point at 1 and we have

$$
b^{n}(0) \rightarrow 1 \text { as } n \rightarrow \infty \text { and } b^{n}(0) \geq 5 / 6 \text { for } n \in \mathbb{N},
$$

and so

$$
\operatorname{dist}\left(\varphi^{\ell_{n}}(4), \partial G_{n}\right) \rightarrow 0 \text { as } n \rightarrow \infty, \quad \text { and } \quad \varphi^{\ell_{n}}(4)-\kappa_{n} \geq 5 / 6 \quad \text { for } n \in \mathbb{N} \text {. }
$$


We also note that if $0 \leq z_{1}, z_{2}<1$, then

$$
\left|b\left(z_{1}\right)-b\left(z_{2}\right)\right|=\left|\frac{11\left(z_{1}-z_{2}\right)}{\left(6+5 z_{1}\right)\left(6+5 z_{2}\right)}\right| \leq \frac{11\left|z_{1}-z_{2}\right|}{36} .
$$

Next, we take a point $x \in D\left(4, r_{0}\right)$ such that $f(x)=\kappa_{0}$, which is possible by Theorem 3.1(i), and consider the orbit of $x$ under iteration by $f$. We claim that, for $n \geq 0$,

$$
\left|f^{\ell_{n}}(x)-\varphi^{\ell_{n}}(4)\right| \leq \sum_{i=1}^{n} \alpha_{i}\left(\frac{11}{36}\right)^{n-i} \leq \frac{1}{2^{n}} .
$$

We prove (5.19) by induction. First, we note that it is true if $n=0$, since $f(x)-$ $\varphi(4)=0$. Next, suppose that (5.19) holds for $n=m \geq 0$. Then it follows from (5.17) and (5.19) that $f^{\ell_{m}}(x)>0$, and so it follows from Lemma 4.1(b) together with (5.18) that, for $m \geq 0$,

$$
\begin{aligned}
\left|f^{\ell_{m+1}}(x)-\varphi^{\ell_{m+1}}(4)\right| & \leq \alpha_{m+1}+\left|b_{m}\left(f^{\ell_{m}}(x)-\kappa_{m}\right)-b_{m}\left(\varphi^{\ell_{m}}(4)-\kappa_{m}\right)\right| \\
& \leq \alpha_{m+1}+\frac{11\left|f^{\ell_{m}}(x)-\varphi^{\ell_{m}}(4)\right|}{36} \\
& \leq \alpha_{m+1}+\sum_{i=1}^{m} \alpha_{i}\left(\frac{11}{36}\right)^{m+1-i} \\
& =\sum_{i=1}^{m+1} \alpha_{i}\left(\frac{11}{36}\right)^{m+1-i} \\
& \leq \sum_{i=1}^{m+1} \frac{1}{6^{i}}\left(\frac{11}{36}\right)^{m+1-i} \leq \frac{m+1}{3^{m+1}} \leq \frac{1}{2^{m+1}} .
\end{aligned}
$$

Thus (5.19) holds for $n=m+1$ and hence, by induction, for all $n \geq 0$.

It follows from (5.19) together with (5.17) that

$$
\operatorname{dist}\left(f^{\ell_{n}}(s), \partial G_{n}\right) \rightarrow 0 \quad \text { as } n \rightarrow \infty
$$

and so, by Lemma 5.4(b), orbits of points in $U_{0}$ behave as described in Theorem 5.2(c).

Acknowledgements. We would like to thank Anna Miriam Benini, Chris Bishop, Nuria Fagella and Lasse Rempe for inspiring discussions. All authors were supported by Engineering and Physical Sciences Research Council grant EP/R010560/1.

\section{REFERENCES}

[Bak76] I. N. Baker. An entire function which has wandering domains. J. Aust. Math. Soc. 22(2) (1976), 173-176.

[Bak84] I. N. Baker. Wandering domains in the iteration of entire functions. Proc. Lond. Math. Soc. (3) 49(3) (1984), 563-576.

[BEF+21] A. M. Benini, V. Evdoridou, N. Fagella, P. Rippon and G. Stallard. Classifying simply connected wandering domains. Math. Ann. (2021), doi:10.1007/s00208-021-02252-0.

[Ber93] W. Bergweiler. Iteration of meromorphic functions. Bull. Amer. Math. Soc. 29(2) (1993), 151-188. 
[Bis15] C. J. Bishop. Constructing entire functions by quasiconformal folding. Acta Math. 214(1) (2015), $1-60$.

[Boc21] L. Boc Thaler. On the geometry of simply connected wandering domains. Bull. Lond. Math. Soc. 53(6) (2021), 1663-1673.

[BRS13] W. Bergweiler, P. J. Rippon and G. M. Stallard. Multiply connected wandering domains of entire functions. Proc. Lond. Math. Soc. (3) 107(6) (2013), 1261-1301.

[Dev90] R. L. Devaney. Dynamics of entire maps. Workshop on Dynamical Systems (Trieste, 1988) (Pitman Research Notes in Mathematics Series, 221). Longman Scientific \& Technical, Harlow, 1990, pp. 1-9.

[dMvS89] W. de Melo and S. van Strien. A structure theorem in one-dimensional dynamics. Ann. of Math. (2) 129(3) (1989), 519-546.

[EL87] A. E. Eremenko and M. Ju. Ljubich. Examples of entire functions with pathological dynamics. J. Lond. Math. Soc. (2) 36(3) (1987), 458-468.

[FH06] N. Fagella and C. Henriksen. Deformation of entire functions with Baker domains. Discrete Contin. Dyn. Syst. 15(2) (2006), 379-394.

[FJL19] N. Fagella, X. Jarque and K. Lazebnik. Univalent wandering domains in the Eremenko-Lyubich class. J. Anal. Math. 139(1) (2019), 369-395.

[Her84] M. R. Herman. Exemples de fractions rationnelles ayant une orbite dense sur la sphère de Riemann. Bull. Soc. Math. France 112(1) (1984), 93-142.

[MPS20] D. Martí-Pete and M. Shishikura. Wandering domains for entire functions of finite order in the Eremenko-Lyubich class. Proc. Lond. Math. Soc. (3) 120(2) (2020), 155-191.

[Sul85] D. Sullivan. Quasiconformal homeomorphisms and dynamics. I. Solution of the Fatou-Julia problem on wandering domains. Ann. of Math. (2) 122(3) (1985), 401-418. 